\title{
The onset of thermal convection in Ekman-Couette shear flow with oblique rotation
}

\author{
By Y. PONTY', A. D. GILBERT ${ }^{2}$ AND A. M. SOWAR D \\ ${ }^{1}$ Observatoire de la Cote d'Azur - Nice, Laboratoire Cassini - CNRS UMR 6529, \\ BP 4229, 06304 Nice Cedex 4, France \\ ${ }^{2}$ School of Mathematical Sciences, University of Exeter, Exeter EX4 4QE, UK
}

(Received 17 June 2002 and in revised form 27 November 2002)

The onset of convection of a Boussinesq fluid in a horizontal plane layer is studied. The system rotates with constant angular velocity $\boldsymbol{\Omega}$, which is inclined at an angle $\vartheta$ to the vertical. The layer is sheared by keeping the upper boundary fixed, while the lower boundary moves parallel to itself with constant velocity $\boldsymbol{U}_{0}$ normal to the plane containing the rotation vector and gravity $\boldsymbol{g}$ (i.e. $\boldsymbol{U}_{0} \| \boldsymbol{g} \times \boldsymbol{\Omega}$ ). The system is characterized by five dimensionless parameters: the Rayleigh number $R a$, the Taylor number $\tau^{2}$, the Reynolds number $R e$ (based on $\boldsymbol{U}_{0}$ ), the Prandtl number $\operatorname{Pr}$ and the angle $\vartheta$. The basic equilibrium state consists of a linear temperature profile and an Ekman-Couette flow, both dependent only on the vertical coordinate $z$. Our linear stability study involves determining the critical Rayleigh number $R a_{c}$ as a function of $\tau$ and $R e$ for representative values of $\vartheta$ and $P r$.

Our main results relate to the case of large Reynolds number, for which there is the possibility of hydrodynamic instability. When the rotation is vertical $\vartheta=0$ and $\tau \gg 1$, so-called type I and type II Ekman layer instabilities are possible. With the inclusion of buoyancy $R a \neq 0$ mode competition occurs. On increasing $\tau$ from zero, with fixed large $R e$, we identify four types of mode: a convective mode stabilized by the strong shear for moderate $\tau$, hydrodynamic type I and II modes either assisted $(R a>0)$ or suppressed $(R a<0)$ by buoyancy forces at numerically large $\tau$, and a convective mode for very large $\tau$ that is largely uninfluenced by the thin Ekman shear layer, except in that it provides a selection mechanism for roll orientation which would otherwise be arbitrary. Significantly, in the case of oblique rotation $\vartheta \neq 0$, the symmetry associated with $\boldsymbol{U}_{0} \leftrightarrow-\boldsymbol{U}_{0}$ for the vertical rotation is broken and so the cases of positive and negative $R e$ exhibit distinct stability characteristics, which we consider separately. Detailed numerical results were obtained for the representative case $\vartheta=\pi / 4$. Though the overall features of the stability results are broadly similar to the case of vertical rotation, their detailed structure possesses a surprising variety of subtle differences.

\section{Introduction}

Thermal convection and shear flow instability are pervading themes in fluid dynamic stability theory. The additional influence of rotation is a key ingredient in the study of astrophysical and geophysical fluid motion. There has been considerable progress made in our understanding of both the onset of convection via linear instability and the ensuing finite-amplitude convection. Though many of the ingredients 
mentioned - rotation, shear and thermal gradients - have been studied in isolation or in particular combinations, there has been no comprehensive investigation of even linear stability with all possible combinations of the many dimensionless parameters that characterize such a system. Our present linear investigation is motivated by our dynamo models (Ponty, Gilbert \& Soward 2001a,b) which build upon the results that we present here. These dynamo studies relate to magnetic field generation in the tachocline of the Sun and we will expand on the geometry below. It is sufficient to note here that our work certainly does not encompass all basic configurations but does build upon, link and extend extensive earlier results.

We begin by reviewing the basic ideas related to the stability of fluid confined between two parallel planes of infinite horizontal extent. In the absence of shear and rotation, static Boussinesq fluid heated from below and subject to a linear adverse temperature gradient becomes unstable to small perturbations when the Rayleigh number $R a$ exceeds some critical value $R a_{c 0}$. Onset of convection is characterized by steady convective rolls. Their orientation is identified by a horizontal wave vector $\boldsymbol{k}$ normal to the roll axis. In view of the rotational symmetry of the system about the vertical, the direction of $\boldsymbol{k}$ is arbitrary. Nevertheless, with the addition of shear or inclined rotation this isotropy is broken and a preferred roll orientation emerges.

In the absence of rotation, a unidirectional shear flow may be generated either by moving the rigid boundaries parallel to themselves (plane Couette flow with a linear profile) or by keeping the boundaries fixed and applying a constant pressure gradient (plane Poiseuille flow with a parabolic profile), or a combination of both. Without buoyancy forces plane Couette flow is always stable in the linear approximation, whereas when the Reynolds number $R e$ is sufficiently large, plane Poiseuille flow becomes unstable to travelling transverse rolls (Tollmien-Schlicting waves) with their wave vector aligned with the mean flow. With the addition of buoyancy, as described in the classical Rayleigh-Bénard problem above, the preferred mode for Poiseuille flow is purely convective for small $R e$. It takes the form of longitudinal rolls which do not interact with the shear and occur when the Rayleigh number takes the critical value $R a_{c 0}$ mentioned above. The anisotropy introduced by the shear only selects the roll orientation for convection. Nevertheless, once $R e$ reaches a value close to the value for pure hydrodynamic instability for which $R a_{c}=0$, the transverse rolls of TollmienSchlicting type take over (see e.g. Gage \& Reid 1968). Longitudinal convective rolls are appropriate to the corresponding Couette flow problem at all values of $R e$ (see e.g. Ingersoll 1965; Clever, Busse \& Kelly 1977). Various developments of this theme are discussed in the review article of Kelly (1994).

With the addition of rotation, measured by the Taylor number $\tau^{2}$, the situation becomes more complicated. Kropp \& Busse (1991) considered thermal convection in plane Couette flow with rotation horizontal and transverse to the shear. Two types of mode can be distinguished. On the one hand, hydrodynamic instability of the plane Couette flow is manifested as Taylor vortices which are longitudinal rolls. On the other, transverse rolls are driven by buoyancy forces as would occur in the absence of shear. Since they do not vary in the direction of the rotation vector, they are unaffected by the Coriolis force and so occur when the Rayleigh number takes the critical value $R a_{c 0}$ mentioned above. Once all these ingredients occur simultaneously, the longitudinal and transverse rolls compete with each other. Which of the two is preferred is clearly mapped out by Kropp \& Busse (1991) in their figure 2. It is also important to note that the critical Rayleigh number has a complicated dependence on $\tau$ and $R e$. That work embraces an important special case of the geometry that we investigate. 
We remark briefly on other closely related orientations of the rotation vector $\boldsymbol{\Omega}$ that only loosely impinge on our investigations. Busse \& Kropp (1992) considered the case of aligned horizontal rotation and shear, and found that, paradoxically, buoyantly driven oblique rolls are sometimes preferred. Matthews \& Cox (1997) extended these studies by allowing an arbitrary angle between the horizontal rotation vector and the linear shear flow. They studied the roll orientation as a function of this angle and used arguments based on winding numbers to determine the dominant driving mechanism for the rolls, shear or convection, in certain parameter ranges.

When the rotation vector has a vertical component $\boldsymbol{\Omega}_{v}$, a shear flow driven by a horizontal pressure gradient or by moving rigid boundaries is no longer unidirectional. Indeed in the limit of strong rotation $\tau \gg 1$, the flow is confined to Ekman boundary layers which are characterized by Ekman spirals. In addition to the pure hydrodynamic instabilities associated with this spiralling flow, this extra structure also complicates the convective instability.

Our study is motivated by an astrophysical application, namely to find a simple fluid dynamical model of the layer at the base of the Solar convection zone known as the 'tachocline' (Spiegel \& Zahn 1992). This zone is believed to play an important role in the mixing between the radiative and the convective zones of the sun and may also be the principal seat of the Solar dynamo (see Weiss 1994 for a review). In the tachocline there is strong shear from the differential rotation, which can lead to the $\omega$-effect in kinematic dynamo theory, and helical overshooting convection should generate an $\alpha$-effect, leading to travelling $\alpha \omega$-dynamo waves. Note that according to helioseismological results (Dziembowski, Goode \& Libbrecht 1989) the sense of the shear in the tachocline varies with latitude. Near the poles the radiative interior rotates faster than the convection zone: the vorticity in the shear flow is then in the opposite sense to the rotation of the whole system (corresponding to $R e>0$ in the framework given below). At the equator the vorticity is aligned (corresponding to $R e<0$ ). Thus the susceptibility of the shear layer to convective or hydrodynamic instabilities varies significantly from poles to equator.

To this end we study the convective instability of a shear flow in a rotating plane layer, which models local Cartesian geometry in the northern hemisphere of a spherical shell at a co-latitude $\vartheta$. The rotation vector has a horizontal component $\boldsymbol{\Omega}_{h}$ in the northerly direction provided $\vartheta \neq 0$, i.e. except at the north pole. For reasons which we expand upon elsewhere (Ponty et al. 2001a), we adopt a reference frame moving locally with the convection zone relative to which the radiative zone becomes a moving boundary. Our shear is thus generated by the motion of that lower rigid boundary in the easterly direction with velocity $\boldsymbol{U}_{0}$, perpendicular to the rotation vector $\boldsymbol{\Omega}$ and gravity $\boldsymbol{g}$ such that $\boldsymbol{U}_{0} \| \boldsymbol{g} \times \boldsymbol{\Omega}$. The upper boundary is fixed, and there is no imposed pressure gradient. By this device, in the large-Taylor-number limit $\tau^{2} \gg 1$ the shear flow takes the form of an Ekman layer localized in the vicinity of the lower boundary, while the fluid is almost stationary elsewhere. Though it goes beyond the scope of this paper to attempt to model the detailed physics of the tachocline, this application motivates our study of convection in a rotating shear flow.

Our model combines several important physical effects: unstable stratification (buoyancy), horizontal rotation $\boldsymbol{\Omega}_{h}$ (for $\vartheta \neq 0$ ), vertical rotation $\boldsymbol{\Omega}_{v}$ (for $\vartheta \neq \pi / 2$ ), and shear in the form of an Ekman layer for non-zero vertical rotation $\left(\left|\boldsymbol{\Omega}_{v}\right| \neq 0\right)$, or a linear plane Couette shear for purely horizontal rotation $\left(\left|\boldsymbol{\Omega}_{v}\right|=0\right.$, as considered by Kropp \& Busse 1991) appropriate at the equator, $\vartheta=\pi / 2$. Our aim is to distinguish the roles played by these physical ingredients in determining the nature of the onset of convective and/or hydrodynamic instability. The particular characteristics of interest 
are the critical values of wavenumber $\left|\boldsymbol{k}_{c}\right|$, the orientation of the convective roll axis $\boldsymbol{g} \times \boldsymbol{k}_{c}$, the frequency $\omega_{c}$ and Rayleigh number $R a_{c}$ for the onset of instability. Though we only explore linear stability, this is a necessary first step before considering the weakly and strongly nonlinear regimes. Indeed the results presented here form the basis of the kinematic dynamo calculations undertaken by Ponty et al. (2001a).

A study of hydrodynamic instabilities (zero Rayleigh number: $R a=0$ ) in a rotating layer with velocities $\bar{\mp} \boldsymbol{U}_{0} / 2$ imposed on the top and bottom surfaces was initiated by Hoffman, Busse \& Chen (1998), who considered the case of vertical rotation $\left(\left|\boldsymbol{\Omega}_{h}\right|=0\right)$ appropriate at the north pole $\vartheta=0$. Unlike our problem the basic flow has an Ekman layer at both the top and bottom boundaries in the large rotation limit, whereas we have just an Ekman layer at the bottom boundary, which carries a net Ekman flux. These distinct flows arise because the pressure gradient is not Galilean invariant in a rotating reference frame, as discussed further in Ponty et al. (2001a). Indeed, Hoffmann \& Busse (2001) have extended their study by including an applied constant horizontal pressure gradient perpendicular to the direction of the moving boundaries (i.e. $-\nabla p \| 2 \boldsymbol{\Omega} \times \boldsymbol{U}_{0}$ ). Their problem reduces to ours for the special case $\nabla p=\boldsymbol{\Omega} \times \boldsymbol{U}_{0}$, for which their mainstream velocity outside boundary layers takes the upper boundary value $-\boldsymbol{U}_{0} / 2$ in the large-Taylor-number limit, $\tau \gg 1$. None of the results reported by them are for this case of negative mainstream velocity.

Hoffman \& Busse (1999) extended the Hoffman et al. (1998) analysis of hydrodynamic instability to the case of oblique rotation $(\vartheta \neq 0)$, which like ours has the shear perpendicular to it: $\boldsymbol{U}_{0} \perp \boldsymbol{\Omega}$. That enables them to identify simultaneously the so-called type I and type II Ekman layer instabilities associated with vertical rotation $\left(\left|\boldsymbol{\Omega}_{v}\right| \neq 0\right)$ as well as the Taylor vortices associated with plane Couette flow, which occurs when the rotation is horizontal $\left(\left|\boldsymbol{\Omega}_{v}\right|=0\right)$ at $\vartheta=\pi / 2$. Prior to these studies, Cox (1998) investigated convective instabilities in the same geometry, although his rotation vector $\boldsymbol{\Omega}$ is arbitrarily orientated albeit our case $\boldsymbol{U}_{0} \| \boldsymbol{g} \times \boldsymbol{\Omega}$ occurs as a special case. Since he employed relatively low values of the Reynolds number $R e$, he only found convective types of critical modes with $R a_{c}>0$, but pursued the numerical simulation of them into the nonlinear regime. In making detailed comparisons with the Hoffman et al. (1998) and Hoffman \& Busse (1999) studies, it is important to note that Cox (1998) adopts a reversed sign for the top and bottom surfaces, namely $\pm \boldsymbol{U}_{0} / 2$. Indeed our lower-boundary velocity $\boldsymbol{U}_{0}$ is closely linked to the former convention in the large-Taylor-number limit as we explained in the paragraph above.

Our paper is organized as follows. In $\S 2$ we formulate the equations governing convection in the rotating, sheared plane layer, and set up a Cartesian coordinate system based on the roll axis of the linearly unstable mode. In $\S 3$ we consider the case of vertical rotation, $\vartheta=0$. We focus on values of the Reynolds number Re sufficiently large to have the possibility of driving pure hydrodynamic instabilities, which in the large- $\tau$ limit are the type I and type II Ekman layer instabilities. Our principal concern is with the competition between these hydrodynamic modes and the pure convective modes. On seeking the critical Rayleigh number $R a_{c}$ for the onset of instability, we find that the preferred modes are of convective type for small and large $\tau$, while for sufficiently large $R e$ and for intermediate values of $\tau$ the preferred modes are of Ekman type I or II. In $\S 4$ we consider the case of oblique rotation $\vartheta \neq 0$. We find that the stability characteristics at mid-latitudes resemble the vertical rotation case. The main differences stem from the fact that there is now no longer a symmetry associated with changing the direction of the movement of the bottom boundary from 


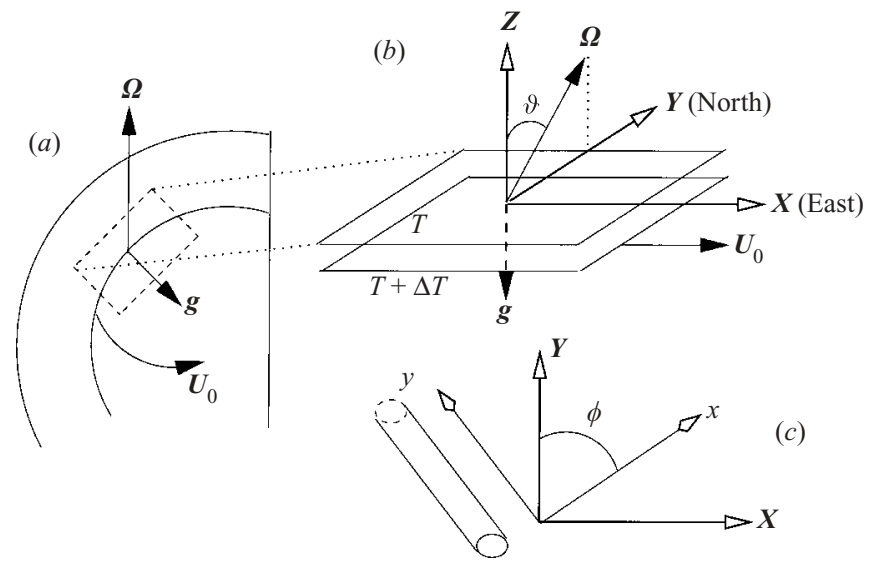

FiguRE 1. (a) The spherical geometry relevant to the Solar dynamo. The dashed box indicates schematically a local Cartesian approximation at a co-latitude $\vartheta$, which includes part of the convection zone and overshoot layer. $(b)$ The plane layer system. The gravitational acceleration $\boldsymbol{g}$ is in the $(-z)$-direction; the rotation vector $\boldsymbol{\Omega}$ lies in the, $(Y, z)$-plane and makes an angle $\vartheta$ with the vertical; a velocity $\boldsymbol{U}_{0}$ in the $X$-direction is imposed at the base of the layer (and zero velocity at the top); the fluid layer is heated from below. (c) The relation between the $x, y$ and $X, Y$-axes. The roll axes are in the $y$-direction; the wave vector $\boldsymbol{k}$ points in the $x$-direction.

eastwards to westwards (i.e. $\boldsymbol{U}_{0}$ and $-\boldsymbol{U}_{0}$ are not equivalent). We therefore discuss the cases $R e>0$ and $R e<0$ separately. Indeed this lack of symmetry is the reason why we stressed above that Cox (1998) essentially adopts a different sign convention to us. We also take some care in $\S 4.1$ to distinguish the various hydrodynamic Ekman layer and Taylor vortex instabilities that might occur at low latitudes close to the equator $\vartheta \simeq \pi / 2$. We conclude with a brief discussion of our results in $\S 5$.

Before continuing we remark that there is an extensive literature concerning stellar convection as well as convection in the giant planets and in the Earth's planetary boundary layer. For example, Hathaway, Toomre \& Gilman (1980) and Hathaway \& Somerville (1986) considered the stability of a sheared convective flow at any latitude, i.e. with the rotation vector at any angle $\vartheta$ to the horizontal. However, to maintain a unidirectional shear flow (not spiralling) they invoked additional body forces such as a north-south temperature gradient which leads to a thermal wind in the eastwest direction. In this configuration they found that convective rolls may be aligned north-south or east-west, depending on the parameter values characterizing the system. The interaction of Ekman instabilities and convection or stable stratification is also important in the planetary boundary layer (see Etling 1971; Brown 1972; Asai \& Nakasuji 1973; Wipperman, Etling \& Kirstein 1978; also the review Etling \& Brown 1993).

\section{Governing equations}

In this section, we provide the mathematical formulation of our problem. In $\S 2.1$, we non-dimensionalize our equations and identify the principal dimensionless parameters. A two-dimensional representation is developed in $\S 2.2$. This simplification anticipates the nature of the linear instabilities, whose governing equations are obtained in $\S 2.3$.

\subsection{Locally Cartesian formulation}

Our horizontal plane layer model is motivated by the problem of Solar convection with shear and rotation in the spherical geometry sketched in figure 1(a). At a 
co-latitude $\vartheta$, we adopt a local Cartesian approximation with coordinates $(X, Y, z)$ as shown in figure $1(b)$. The $z$-axis is vertically upwards, while the associated (constant) gravity $\boldsymbol{g}=-g \widehat{\boldsymbol{z}}$ is downwards (here and below the hat is used to denote unit vectors). The $Y$-axis points northwards so that the rotation vector $\Omega$ lies in the $(Y, z)$-plane and makes an angle $\vartheta$ with the vertical. We write

$$
\boldsymbol{\Omega}(=\Omega \widehat{\boldsymbol{\Omega}})=\Omega \cos \vartheta \widehat{z}+\Omega \sin \vartheta \widehat{\boldsymbol{Y}} \equiv \boldsymbol{\Omega}_{v}+\boldsymbol{\Omega}_{h},
$$

where $\boldsymbol{\Omega}_{v}=\Omega \cos \vartheta \widehat{z}$ and $\boldsymbol{\Omega}_{h}=\Omega \sin \vartheta \widehat{\boldsymbol{Y}}$ are the vertical and horizontal components of the rotation vector respectively.

Our layer is unbounded in the horizontal $(X, Y)$-plane and is confined vertically between rigid boundaries at $z=0$ and $z=h$. At the top of the layer $z=h$ the fluid velocity vanishes $\boldsymbol{U}=\mathbf{0}$, while the bottom rigid boundary $z=0$ moves parallel to itself steadily eastwards in the $X$-direction $\boldsymbol{U}=\boldsymbol{U}_{0}=U_{0} \widehat{\boldsymbol{X}}$, as shown on figure $1(b)$. In the Solar context, the velocity difference between the base and the top of the layer is chosen to model the difference in angular velocity between the convection zone and the radiative interior, as revealed by helioseismological observations. Note that this geometry requires the velocity $\boldsymbol{U}_{0}$ to be perpendicular to both $\boldsymbol{\Omega}$ and $\boldsymbol{g}$ (i.e. $\boldsymbol{U}_{0} \| \boldsymbol{g} \times \boldsymbol{\Omega}$ ). This feature distinguishes our study from Matthews \& Cox (1997) and Cox (1998), who permit an arbitrary angle between $\boldsymbol{U}_{0}$ and $\boldsymbol{\Omega}_{h}$ (i.e. $\boldsymbol{\Omega} \cdot \boldsymbol{U}_{0} \neq 0$ in general).

Associated with the form of our rotation vector (2.1), the stability characteristics of our system are invariant under two distinct simultaneous transformations involving the reflection $\vartheta \leftrightarrow \pi-\vartheta$ in the equatorial plane: they are $\Omega \leftrightarrow \Omega, U_{0} \leftrightarrow U_{0}$ and $\Omega \leftrightarrow-\Omega, U_{0} \leftrightarrow-U_{0}$. The triple product $\mathscr{T} \equiv \boldsymbol{U}_{0} \cdot(\boldsymbol{g} \times \boldsymbol{\Omega})$ is invariant under both transformations. Only at the poles $\vartheta=0$ and $\pi$, where the rotation vector is vertical and $\mathscr{T}=0$, are the stability characteristics invariant to reversing the sign of $U_{0}$ with all other parameters held fixed. Thus, without loss of generality, we may restrict attention to positive rotation rate $\Omega \geqslant 0$ in the northern hemisphere $0 \leqslant \vartheta \leqslant \pi / 2$, but we must allow $U_{0}$ to take either sign (except at $\vartheta=0$ ). In the context of the Solar convection zone, for which the differential rotation speed is slower near the poles than the equator, the lower boundary velocity $\boldsymbol{U}_{0}$ is eastward $\left(U_{0}>0, \mathscr{T}>0\right)$ near the north pole and westward $\left(U_{0}<0, \mathscr{T}<0\right)$ near the equator.

The upper and lower boundaries are maintained at constant temperatures $T=T_{0}$ and $T=T_{1}$. We assume that the fluid is incompressible, but make the Boussinesq approximation. We take the fluid's kinematic viscosity $\nu$, thermal diffusivity $\kappa$, and coefficient $\alpha$ of expansion to be constant. Under these assumptions the equations of motion in the rotating frame are

$$
\begin{gathered}
\rho_{0}\left(\frac{\partial \boldsymbol{U}}{\partial t}+\boldsymbol{U} \cdot \nabla \boldsymbol{U}+2 \boldsymbol{\Omega} \times \boldsymbol{U}\right)=-\nabla P+\rho \boldsymbol{g}+\rho_{0} \nu \nabla^{2} \boldsymbol{U}, \\
\nabla \cdot \boldsymbol{U}=0, \quad \rho=\rho_{0}\left[1-\alpha\left(T-T_{0}\right)\right], \\
\frac{\partial T}{\partial t}+\boldsymbol{U} \cdot \nabla T=\kappa \nabla^{2} T .
\end{gathered}
$$

The governing equations possess a steady-state solution $\boldsymbol{U}=\boldsymbol{U}_{\mathrm{eq}}(z), T=T_{\mathrm{eq}}(z)$, $P=P_{\text {eq }}(z)$ dependent only on the vertical coordinate $z$. Here heat is transported via vertical conduction alone, while the fluid motion is of Ekman-Couette type with structure

$$
\boldsymbol{U}_{\mathrm{eq}}=U_{0} \boldsymbol{\Lambda}(z), \quad \boldsymbol{\Lambda}=\Lambda_{1}(z) \widehat{\boldsymbol{X}}+\Lambda_{2}(z) \widehat{\boldsymbol{Y}}
$$


The general solution may be expressed in terms of the fluctuations $\boldsymbol{u}, \theta$ and $\Pi$ about this equilibrium state as follows:

$$
\boldsymbol{U}=\boldsymbol{U}_{\mathrm{eq}}(z)+\boldsymbol{u}, \quad T=T_{\mathrm{eq}}(z)+\theta, \quad P=P_{\mathrm{eq}}(z)+\Pi .
$$

We adopt the depth $h$ of our layer as the unit of length and the corresponding diffusion time $h^{2} / \kappa$ as the unit of time. Accordingly we introduce dimensionless variables (primed) defined by

$$
t=t^{\prime} \frac{h^{2}}{\kappa}, \quad \boldsymbol{x}=\boldsymbol{x}^{\prime} h, \quad \boldsymbol{u}=\boldsymbol{u}^{\prime} \frac{\kappa}{h}, \quad \theta=\theta^{\prime} \frac{\nu \kappa}{\alpha g h^{3}}, \quad \Pi=\Pi^{\prime} \frac{\rho_{0} \kappa^{2}}{h^{2}} .
$$

Upon dropping the primes, the governing equations take the dimensionless form

$$
\begin{aligned}
& \frac{1}{P r}\left(\frac{\partial \boldsymbol{u}}{\partial t}+\boldsymbol{u} \cdot \nabla \boldsymbol{u}\right)+\operatorname{Re}\left(\boldsymbol{\Lambda} \cdot \nabla \boldsymbol{u}+\boldsymbol{u} \cdot \widehat{z} \frac{\mathrm{d} \boldsymbol{\Lambda}}{\mathrm{d} z}\right)+\tau \widehat{\boldsymbol{\Omega}} \times \boldsymbol{u}=-\nabla \frac{\Pi}{P r}+\theta \widehat{\boldsymbol{z}}+\nabla^{2} \boldsymbol{u}, \\
& \nabla \cdot \boldsymbol{u}=0 \\
& \frac{\partial \theta}{\partial t}+\boldsymbol{u} \cdot \nabla \theta+\operatorname{Pr} \operatorname{Re} \boldsymbol{\Lambda} \cdot \nabla \theta=\operatorname{Ra} \boldsymbol{u} \cdot \widehat{z}+\nabla^{2} \theta .
\end{aligned}
$$

Here we have introduced dimensionless parameters, namely the Rayleigh number $R a$, the Prandtl number $P r$, the Taylor number $\tau^{2}$ and the Reynolds number $R e$ :

$$
R a=\frac{\alpha g h^{3}\left(T_{1}-T_{0}\right)}{\nu \kappa}, \quad \operatorname{Pr}=\frac{v}{\kappa}, \quad \tau=\frac{2 \Omega h^{2}}{v}, \quad R e=\frac{U_{0} h}{v} .
$$

The isothermal and no-slip boundary conditions are

$$
\boldsymbol{u}=\mathbf{0}, \quad \theta=0 \quad \text { at } \quad z=0 \text { and } 1 .
$$

The underlying Ekman-Couette flow may be expressed in terms of a single dimensionless complex function

$$
\Lambda(z)=\Lambda_{1}(z)+\mathrm{i} \Lambda_{2}(z) \quad \text { on } \quad 0 \leqslant z \leqslant 1,
$$

which in the absence of a horizontal pressure gradient satisfies

$$
\frac{\mathrm{d}^{2} \Lambda}{\mathrm{d} z^{2}}-2 \mathrm{i} \mu^{2} \Lambda=0, \quad \text { where } \quad \mu=\sqrt{\frac{\tau \cos \vartheta}{2}},
$$

together with the boundary conditions

$$
\Lambda(0)=1, \quad \Lambda(1)=0 .
$$

The solution

$$
\Lambda=-\frac{\sinh [(1+\mathrm{i}) \mu(z-1)]}{\sinh [(1+\mathrm{i}) \mu]} \quad \text { for } \quad \mu \neq 0
$$

yields the family of Ekman-Couette flows with components

$$
\begin{aligned}
& \Lambda_{1}=\frac{\cosh [\mu(z-2)] \cos (\mu z)-\cosh (\mu z) \cos [\mu(z-2)]}{\cosh (2 \mu)-\cos (2 \mu)}, \\
& \Lambda_{2}=\frac{\sinh [\mu(z-2)] \sin (\mu z)-\sinh (\mu z) \sin [\mu(z-2)]}{\cosh (2 \mu)-\cos (2 \mu)}
\end{aligned}
$$

an example is provided in figure 2. When the vertical component of rotation is small, 

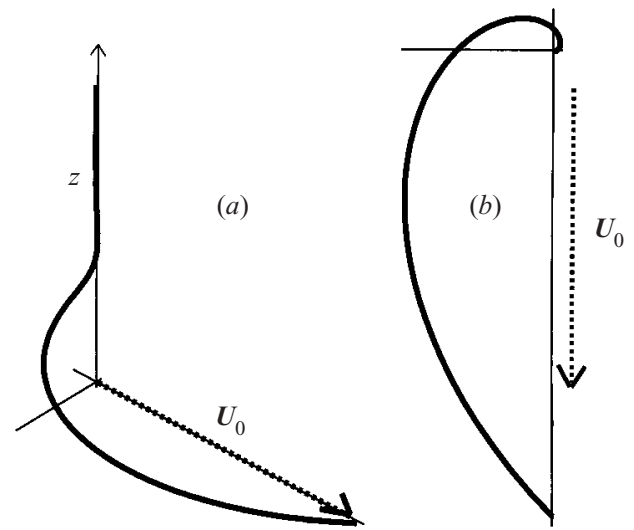

FIgURE 2. The Ekman spiral. (a) The full line gives a perspective view of the Ekman layer velocity, given by plotting $\left(\Lambda_{1}(z), \Lambda_{2}(z), z\right)$ as $z$ varies between 0 and 1 , for $\tau=100$. The dotted line represents the velocity $\boldsymbol{U}_{0}$ of the bottom of the plane layer. (b) The same spiral Ekman velocity is projected onto the horizontal $(X, Y)$-plane.

$(2.11 b, c)$ has the power series expansion

$$
\Lambda_{1} \sim 1-z, \quad \Lambda_{2} \sim-\frac{1}{3} \mu^{2} z(1-z)(2-z) \quad \text { for } \quad|\mu| \ll 1,
$$

which reduces to plane Couette flow in the limit $\mu=0$. When the vertical rotation is strong, $(2.11 b, c)$ has the asymptotic representation

$$
\Lambda_{1} \sim \mathrm{e}^{-\mu z} \cos \mu z, \quad \Lambda_{2} \sim-\mathrm{e}^{-\mu z} \sin \mu z \quad \text { as } \quad \mu \rightarrow \infty,
$$

which describes an Ekman boundary layer localized within a distance $O\left(\mu^{-1}\right)$ from the lower boundary; elsewhere the fluid is essentially stationary. In all cases, a southerly (northerly) mass flux of order $\tau^{-1 / 2}$ per unit length ensues when $U_{0}>0\left(U_{0}<0\right)$. In the Solar context this small flux is returned by very small velocities, in the convection zone high above the thin layer. The up-down asymmetry of our Ekman-Couette flow (2.11) results from our assumption of no horizontal pressure gradient. In the large- $\tau$ limit (2.13) it ensures that there is no geostrophic motion outside the bottom Ekman layer. Of course other choices of uniform geostrophic flow corresponding to finite pressure gradients are possible, as considered by Hoffmann \& Busse (2001), but the present choice provides the most faithful representation of the Solar tachocline (a single layer in the boxed region of figure 1a) as Ponty et al. (2001a) explain in some detail (see also Ponty et al. 2001b). It is important to appreciate that a horizontal pressure gradient is not Galilean invariant in a rotating system. So if we had adopted coordinates for which the bottom boundary was at rest with the geostrophic flow $-\boldsymbol{U}_{0}$ above, there would be a finite pressure gradient in that reference frame.

In our construction, we have implicitly assumed that $U_{0}$ is positive in defining the Reynolds number, while the vector field $\boldsymbol{\Lambda}$ has been normalized via the boundary condition $(2.10 c)$. Since both signs of $U_{0}$ are relevant in the Sun - positive $U_{0}>0$ near the poles and negative $U_{0}<0$ near the equator - we find it convenient to retain the boundary condition $\Lambda(0)=1$ but permit the Reynolds number $R e$ to take positive and negative values in concert with $U_{0}$. Interestingly, when $\left|\boldsymbol{\Omega}_{h}\right| \neq 0$ the stability characteristics depend on the sign of $R e$. This is because the absolute vorticity in the non-rotating inertial frame is different in the two cases, i.e. the vorticity of the shear either reinforces or weakens the vorticity $2 \Omega$ of the rotating frame. 


\subsection{Two-dimensional formulation}

In the case of purely horizontal rotation $\left(\left|\boldsymbol{\Omega}_{v}\right|=0\right)$ at $\vartheta=\pi / 2$ and no shear $R e=0$, the axes of the convection rolls at the onset of instability are aligned with the horizontal rotation vector $\boldsymbol{\Omega}_{h}$, namely the $Y$-direction. In contrast, when there is shear $R e \neq 0$ but no rotation, $\tau=0$, the roll axes of the convection are aligned with the linear shear parallel to $\boldsymbol{U}_{0}$, namely the $X$-direction. These trivial results are well known. Matthews \& Cox (1997) have shown that for the non-trivial extension when both horizontal rotation $\tau \neq 0(\mu=0)$ at $\vartheta=\pi / 2$ and plane Couette shear $(R e \neq 0)$ are present, the onset of convection continues to be characterized by either $X$ - or $Y$-directed rolls.

When, however, there is a vertical component of rotation $\left(\left|\boldsymbol{\Omega}_{v}\right| \neq 0\right)$, the laminar flow loses the uni-directional plane Couette form (2.12) with $\mu=0$, and instead takes on the spiralling Ekman-Couette form $(2.11 b, c)$ appropriate to $\mu \neq 0$. Now the convective roll axes at onset are generally oblique and not aligned with either the $X$ - or $Y$-axes. For this reason, we introduce new horizontal coordinates $(x, y)$ which are related to the roll axes, as shown in figure 1(c). Specifically, since our basic conductive state depends only on the vertical coordinate $z$, we may seek solutions to the linear stability problem that are separable in the horizontal coordinates $X, Y$ and proportional to $\exp \left[\mathrm{i}\left(k_{1} X+k_{2} Y\right)+(\sigma+\mathrm{i} \omega) t\right]$, where the wave vector $\boldsymbol{k}=k_{1} \widehat{\boldsymbol{X}}+k_{2} \widehat{\boldsymbol{Y}}$ is constant and $\sigma+\mathrm{i} \omega$ is the complex growth rate. To simplify our analysis, we choose new horizontal axes such that the $x$-axis is aligned with the wave vector $\boldsymbol{k}$. As a result our separable modes are proportional to $\exp [\mathrm{i} k x+(\sigma+\mathrm{i} \omega) t]($ see $(2.20))$, where $k=|\boldsymbol{k}|$, so that now the roll axes are aligned with the $y$-direction.

We introduce the angle $\phi$ between the wave vector $\boldsymbol{k}$ (or $x$-axis) and the northerly direction (or $Y$-axis); in the spherical shell context, the case $\phi=0$ ( $\boldsymbol{k}$ points northwards) corresponds to doughnut-shaped convection cells, while the case $\phi=\pi / 2$ ( $\boldsymbol{k}$ points eastwards) defines so-called banana-shaped convection cells. With respect to the new $x, y$-axes the horizontal component of rotation makes an angle $\phi$ with the $x$-axis, while the bottom velocity $\boldsymbol{U}_{0}$ subtends an angle $\phi \mp \pi / 2$ with the $x$-axis when $\pm U_{0}>0$ (see figure $1 c$ ). Note that the problem may be formulated equivalently under a rotation of $180^{\circ}$ with $\phi \leftrightarrow \phi+\pi$, provided $k \leftrightarrow-k, \omega \leftrightarrow \omega$. We also have the usual symmetry $\phi \leftrightarrow \phi, k \leftrightarrow-k, \omega \leftrightarrow-\omega$, corresponding to taking the complex conjugate normal mode.

In this new framework the governing equations (2.6) continue to hold, but now the rotation vector (2.1) becomes

$$
\widehat{\boldsymbol{\Omega}}=\sin \vartheta \cos \phi \widehat{\boldsymbol{x}}+\sin \vartheta \sin \phi \widehat{\boldsymbol{y}}+\cos \vartheta \widehat{\boldsymbol{z}},
$$

while the Ekman-Couette flow (2.3) is replaced by

$$
\boldsymbol{\Lambda}=\lambda_{1}(z) \widehat{\boldsymbol{x}}+\lambda_{2}(z) \widehat{\boldsymbol{y}}
$$

where

$$
\lambda_{1}=\Lambda_{1} \sin \phi+\Lambda_{2} \cos \phi, \quad \lambda_{2}=-\Lambda_{1} \cos \phi+\Lambda_{2} \sin \phi .
$$

The advantage of our new coordinates $(x, y, z)$ is that the linear convective mode depends only on the two coordinates $x$ and $z$, while the angle $\phi$ defines the orientation of the rolls. Accordingly, we may introduce the stream function representation for the velocity in the $(x, z)$-plane and write

$$
\boldsymbol{u}(x, z, t)=\nabla \times(\psi \widehat{\boldsymbol{y}})+v \widehat{\boldsymbol{y}} \equiv\left(-\partial_{z} \psi, v, \partial_{x} \psi\right),
$$


where $\partial_{x} \equiv \partial / \partial x$ and $\partial_{z} \equiv \partial / \partial z$. In turn, the vorticity becomes

$$
\nabla \times \boldsymbol{u}=\left(-\partial_{z} v,-\Delta \psi, \partial_{x} v\right), \quad \text { where } \Delta=\partial_{x}^{2}+\partial_{z}^{2} .
$$

We substitute these representations into the $y$-component of the curl of $(2.6 a)$, the $y$-component of $(2.6 a)$ itself and (2.6c). In this way we obtain the nonlinear matrix system

$$
\left(\partial_{t}+\operatorname{Pr} \operatorname{Re} \lambda_{1} \partial_{x}\right) \mathscr{W} \boldsymbol{X}=\mathscr{B} \boldsymbol{X}+\operatorname{Re} \boldsymbol{Y} \partial_{x} \psi-\frac{\partial(\psi, \mathscr{W} \boldsymbol{X})}{\partial(x, z)}
$$

where

$$
\begin{array}{ll}
\boldsymbol{X}=\left[\begin{array}{c}
\psi \\
v \\
\theta
\end{array}\right], & \mathscr{W}=\left[\begin{array}{ccc}
\operatorname{Pr}^{-1} \Delta & 0 & 0 \\
0 & \operatorname{Pr}^{-1} & 0 \\
0 & 0 & 1
\end{array}\right], \\
\boldsymbol{Y}=\left[\begin{array}{c}
\lambda_{1}^{\prime \prime} \\
-\lambda_{2}^{\prime} \\
0
\end{array}\right], & \mathscr{B}=\left[\begin{array}{ccc}
\Delta^{2} & -\tau \partial_{\Omega} & \partial_{x} \\
\tau \partial_{\Omega} & \Delta & 0 \\
R a \partial_{x} & 0 & \Delta
\end{array}\right],
\end{array}
$$

the prime denotes the $z$-derivative $\mathrm{d} / \mathrm{d} z$ and

$$
\partial_{\Omega}=\widehat{\boldsymbol{\Omega}} \cdot \nabla \equiv \cos \vartheta \partial_{z}+\sin \vartheta \cos \varphi \partial_{x} .
$$

The linear operator $\mathscr{B}$ is standard and represents convection in a rotating Boussinesq fluid system. The additional shear flow leads to two new terms. The first one, proportional to $\partial_{x} \mathscr{W} \boldsymbol{X}$ on the left-hand side of the equation, represents the advection of the fluctuating vorticity, momentum and temperature by the mean shear flow $\lambda_{1}$ in the $x$-direction. The term proportional to $\boldsymbol{Y}$ on the right-hand side is a source term representing the advection of vorticity and momentum associated with the mean shear flow $\Lambda$ by the vertical component of the fluctuating velocity $\partial_{x} \psi$.

For our rigid, isothermal boundary conditions, the fluctuation vector $\boldsymbol{X}$ satisfies

$$
\partial_{z} \psi=\psi=v=\theta=0 \quad \text { at } \quad z=0 \text { and } z=1 .
$$

Often, in the absence of shear, stress-free boundary conditions are applied in geophysical applications at small Ekman number to simplify the analysis. It is therefore significant to appreciate that the underlying shear flow in our system can only be maintained by the presence of the moving rigid boundary.

\subsection{Linear stability problem and numerical methods}

We now linearize $(2.18 a)$ with respect to the small disturbances and consider separable modes of the form

$$
\boldsymbol{X}=\tilde{\boldsymbol{X}} \exp [\mathrm{i} k x+(\sigma+\mathrm{i} \omega) t] \quad \text { with } \quad \tilde{\boldsymbol{X}}=[\Psi(z), V(z), \Theta(z)]^{T},
$$

where $\sigma+\mathrm{i} \omega$ is the complex growth rate. The equation for linear perturbations of the $z$-dependent equilibrium state becomes

$$
(\sigma+\mathrm{i} \omega) \widetilde{\mathscr{W}} \widetilde{\boldsymbol{X}}=\widetilde{\mathscr{L}} \widetilde{\boldsymbol{X}}
$$

where

$$
\widetilde{\mathscr{W}}=\left[\begin{array}{ccc}
\operatorname{Pr}^{-1} \widetilde{\Delta} & 0 & 0 \\
0 & \operatorname{Pr}^{-1} & 0 \\
0 & 0 & 1
\end{array}\right]
$$




$$
\widetilde{\mathscr{L}}=\left[\begin{array}{ccc}
\widetilde{\Delta}^{2}-\mathrm{i} k \operatorname{Re} \lambda_{1} \widetilde{\Delta}+\mathrm{i} k \operatorname{Re} \lambda_{1}^{\prime \prime} & -\tau \widetilde{\partial}_{\Omega} & \mathrm{i} k \\
\tau \widetilde{\partial}_{\Omega}-\mathrm{i} k \operatorname{Re} \lambda_{2}^{\prime} & \widetilde{\Delta}-\mathrm{i} k \operatorname{Re} \lambda_{1} & 0 \\
\mathrm{i} k \operatorname{Ra} & 0 & \widetilde{\Delta}-\mathrm{i} k \operatorname{Pr} \operatorname{Re} \lambda_{1}
\end{array}\right]
$$

and

$$
\widetilde{\Delta}=\partial_{z}^{2}-k^{2}, \quad \widetilde{\partial}_{\Omega}=\cos \vartheta \partial_{z}+\mathrm{i} k \sin \vartheta \cos \varphi .
$$

The marginal modes with $\sigma=0$ determine the neutral Rayleigh number $R a$ as a function of the four dimensionless parameters $\operatorname{Pr}, \tau, R e, \vartheta$ of the physical system together with the two mode parameters $k$ and $\phi$. This neutral $R a$ is then to be minimized over all $k$ and $\phi$ to obtain the critical Rayleigh number $\operatorname{R} a_{c}(\operatorname{Pr}, \tau, \operatorname{Re}, \vartheta)$ which occurs at a particular wavenumber $k_{c}$, angle $\phi_{c}$ and frequency $\omega_{c}$.

The system (2.21) is of a generalized eigenvalue form, and was solved in Chebychev spectral space. The tau method was used to implement the rigid boundary conditions. Instead of solving the problem in terms of $\Psi, V$ and $\Theta$ as in (2.21) above, we introduced a fourth variable $\Xi=\widetilde{\Delta} \Psi$ to avoid spurious eigenvalues (see Gottlieb \& Orszag 1977, p. 143). After the calculation of the neutral Rayleigh number for a given $k, \phi$-mode, the main numerical problem is to find the critical Rayleigh number over the $(k, \phi)$-plane using standard minimization methods. The problem becomes numerically difficult when two or more minima occur in this plane (see $\S 3.1$ ), particularly if one of the minima is very narrow (see e.g. figure 6 below).

We will adopt the convention that $k>0$. Accordingly, we need to keep in mind the rotational symmetry, namely that the interchange $\phi \leftrightarrow \phi+\pi$ is accompanied by $\omega \leftrightarrow-\omega$, while $R a$ and $k$ remain unaltered.

\section{Vertical rotation $\vartheta=0$}

Throughout this section we restrict attention to the case of vertical rotation $\left(\left|\boldsymbol{\Omega}_{h}\right|=\right.$ 0 ) at $\vartheta=0$. So in addition to the rotational symmetries already mentioned, our results are invariant under interchanging $U_{0} \leftrightarrow-U_{0}$ (equivalently $R e \leftrightarrow-R e$ ) simultaneously with $\phi \leftrightarrow \phi+\pi$. In $\S 3.1$ we consider only hydrodynamic instability without buoyancy forces $(R a=0)$, while buoyancy related instabilities $(R a \neq 0)$ are discussed in $\$ 3.2$. An asymptotic theory valid in the rapid rotation limit is developed in $\S 3.3$ which gives good agreement with the numerical results for sufficiently large $\tau$.

\subsection{Ekman layer instability $R a=0$}

With rotation vertical $(\vartheta=0)$, the underlying shear flow exhibits the complicated Ekman-Couette profile. When, moreover, the Taylor number $\tau^{2}$ is large, the shear is localized in a thin Ekman layer of width $\mu^{-1}=O\left(\tau^{-1 / 2}\right)$ (see $(2.10 b)$ ) adjacent to the lower boundary, which is characterized by the well-known Ekman spiral (see figure 2). Even without any buoyancy forces $(R a=0)$, the Ekman layer is prone to pure hydrodynamic instabilities at sufficiently large Reynolds number $R e$. These take the form of travelling waves, which destroy the laminar Ekman layer flow.

Ekman layer instabilities have been studied experimentally by Faller (1963) and Caldwell \& Van Atta (1970), and numerically by Faller \& Kaylor (1966) and Lilly (1966). These investigations were concerned with the instability in a semi-infinite plane layer $z \geqslant 0$ of fluid moving steadily with constant velocity $U_{0}$ as $z \rightarrow \infty$, above a rigid stationary boundary at $z=0$. In the absence of a vertical length scale it is natural to adopt the Ekman boundary layer width $D=(v / \Omega)^{1 / 2}$ as the unit of length 

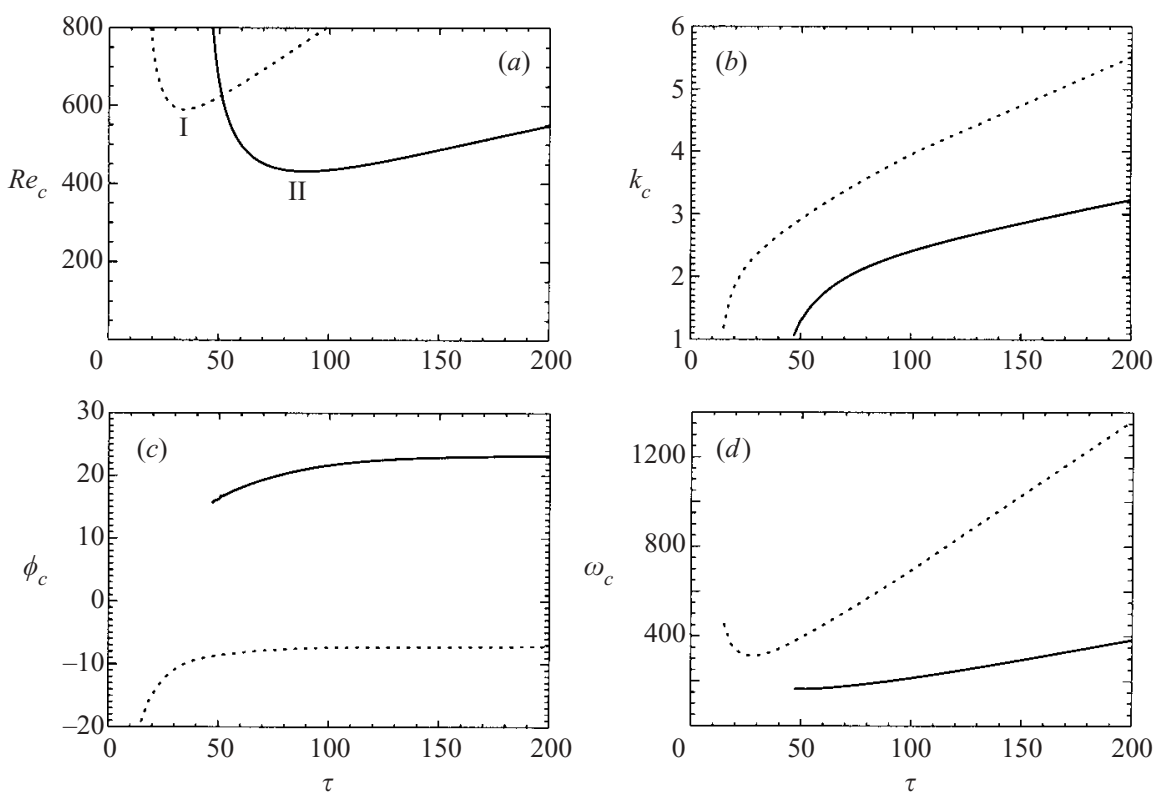

FIGURE 3. The type I (dotted) and type II (solid) modes of the Ekman layer instability. The critical values are plotted against $\tau:(a)$ the Reynolds number $\operatorname{Re}_{c},(b)$ the wavenumber $k_{c}$, (c) the preferred orientation $\phi_{c}$ (in degrees), and $(d)$ the frequency $\omega_{c}$.

and $D / U_{0}$ as the unit of time. The corresponding Reynolds number is

$$
R e^{*}=\frac{D U_{0}}{v}=\frac{U_{0}}{\sqrt{\nu \Omega}}
$$

Two different Ekman layer instabilities are distinguished in these studies; they occur when the Reynolds number $R e^{*}$ exceeds the experimentally measured values of $R e_{c}^{*} \simeq$ 56.7 and 124.5 . The corresponding modes have wavelengths $L_{c}^{*} D$ of roughly $22 D$ and $11 D$ respectively; they have a similar spatial structure but different orientation with respect to the mean flow, with positive and negative $\phi_{c}$ for type II and I respectively. The numerical calculations of Faller \& Kaylor (1966) and Lilly (1966) yielded the critical Reynolds numbers $R e_{c}^{*} \simeq 55$ and 110 in good agreement with the experiments; these instabilities are now referred to as type II and type I respectively.

In our geometry the layer has a finite depth $h$, and so these studies become relevant when $D \ll h$, i.e. for large Taylor number, $\tau^{2} \gg 1$. In this case the Reynolds number based on the Ekman layer thickness may be written as

$$
R e^{*}=\operatorname{Re} \sqrt{2 / \tau}=\operatorname{Re} / \mu \quad(\vartheta=0),
$$

and in our dimensionless units the distance $D$ becomes $\mu^{-1}$, and the time $D / U_{0}$ becomes $\operatorname{Re}^{-1} \mathrm{Pr}^{-1} \mu^{-1}$. We portray our computed values of the critical values $\operatorname{Re}_{c}$, $k_{c}, \phi_{c}$ and $\omega_{c}$ versus $\tau$ for the onset of the Ekman instability in figure 3 .

We observe two modes whose critical values cross around $\tau=50$. For large Taylor number, the instability is linked to the Ekman layer and in that limit the two modes portrayed in figure 3 evidently correspond to the type II mode (solid) and type I mode (dotted). The curves in figure $3(a, b)$ have the asymptotic behaviours $\operatorname{Re}_{c}=R e_{c}^{*} \mu$ and $k_{c}=2 \pi \mu / L_{c}^{*}$ as $\tau \rightarrow \infty$. We fitted our numerical results (not only those depicted but also others at larger values of $\tau$ not shown on the figure) to these power laws, 

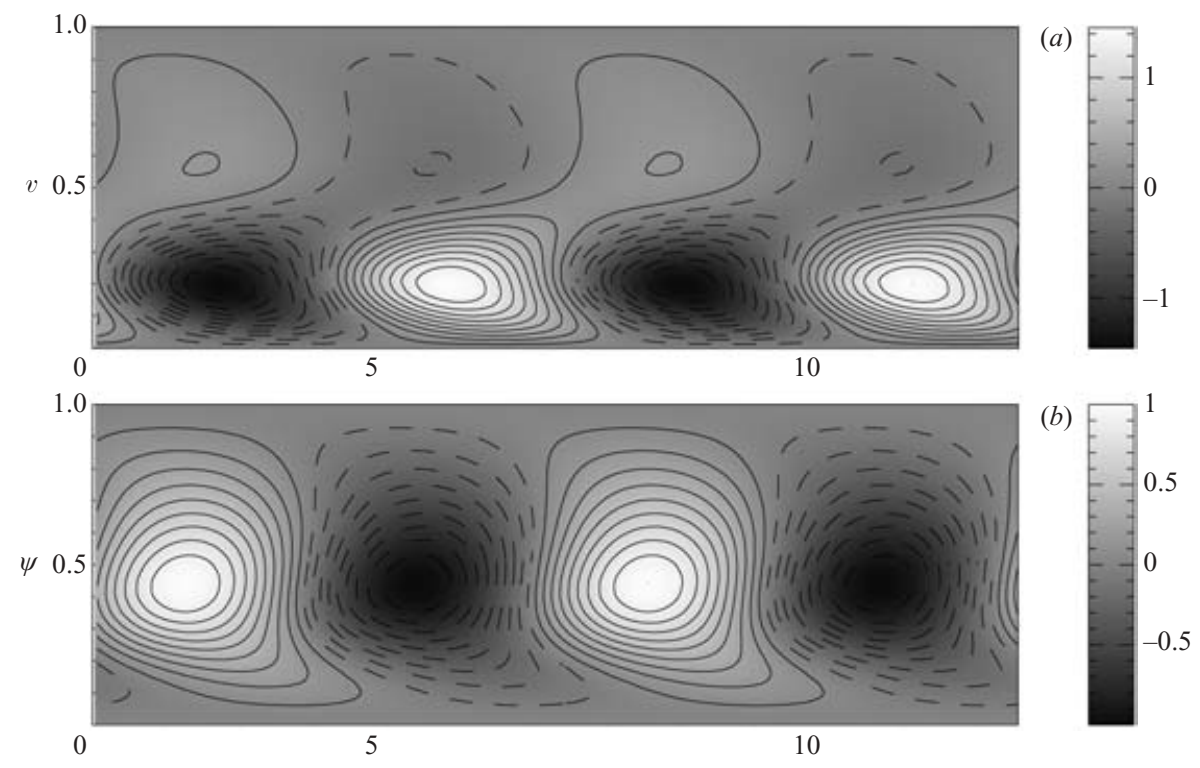

Figure 4. Contour plots of the Couette-Ekman layer type II instability in the $(x+$ $\left.\left(\omega_{c} / k_{c}\right) t, z\right)$-frame co-moving with the wave for $\tau=100, \vartheta=0$. The critical values are $R e_{c} \approx 435.32, k_{c} \approx 2.403, \phi_{c} \approx 21.659^{\circ}$ and $\omega_{c} \approx 214.11$. There is no thermal gradient, $R a=0$. (a) The toroidal velocity $v$, and $(b)$ the stream function $\psi$.

to obtain the values $R e_{c}^{*} \approx 54.21(113.7), L_{c}^{*} \approx 19.9(11.24), \phi_{c}^{*} \approx 23.38^{\circ}\left(-7.3^{\circ}\right)$ and $\omega_{c}^{*} \approx 0.069(0.1220)$ for the solid (dotted) curves, which are clearly correspond well with type II (type I) values. Our critical value $R e_{c}^{*} \approx 54.21$ for type II instability is in particularly good agreement with Iooss, Nielsen \& True (1978) and Melander (1983).

Hoffman et al. (1998) consider a problem similar to ours. It differs in that the upper boundary moves with equal speed but in the opposite direction. So unlike our problem the underlying velocity field is anti-symmetric about the mid-plane, as is also the case in Cox (1998). For large $\tau$ the numerical results listed in Hoffman et al.'s (1998) table 1 compare favourably with ours after the appropriate rescalings. For moderate Taylor numbers some differences are evident between the results portrayed in their figure 4 and ours. On the one hand, the complete type II and type I curves agree qualitatively with our figure 3 . On the other, they isolate a steady mode for small $\tau$ which is not present in our results. The reason is evident from their figure $3(a)$, which shows that the mode has a reflectional symmetry in the mid-plane which our geometry does not admit. Put another way, the type II and I modes are wall modes, while the extra mode of Hoffman et al. (1998) is an interior (centre) mode (called an $S$-mode by Hoffmann \& Busse 2001) linked to the inflection point at the mid-plane.

Figure 4 shows the form of our type II travelling wave instability at large Taylor number $\tau=100$ with $R e_{c} \approx 435.32$. On it, contours of constant toroidal $y$-component of velocity $v$ and poloidal stream function $\psi$ are plotted in the $(x, z)$-plane in the frame co-moving with the wave at its phase velocity $-\omega_{c} / k_{c}$. They are periodic in the $x$-direction with periodicity length $2 \pi / k_{c}$. They illustrate the fact that the toroidal velocity $v$ is activated in the lower Ekman layer and is linked to a poloidal circulation $\psi$ having a return flow outside the Ekman layer. The figure compares well with 

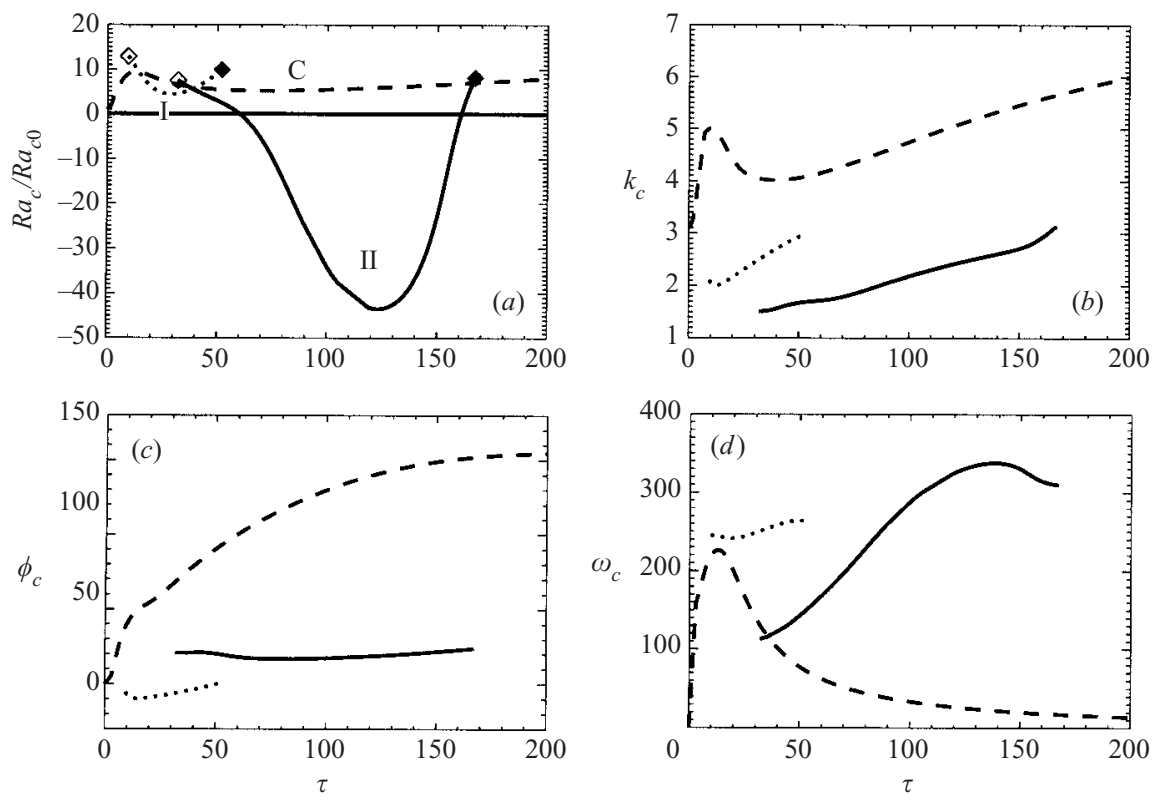

FIGURE 5. The critical values for vertical rotation $\vartheta=0, \operatorname{Pr}=1, R e=500$, plotted against $\tau$. The type I, II and C preferred modes (i.e. the local minima on figure 6) are distinguished by dotted, continuous, and dashed lines respectively and are labelled as such on $(a)$. The approximate locations of the disappearance of the type I and II local minima are designated by the hollow (solid) diamonds on the left (right). The type $\mathrm{C}$ subdivides into $\mathrm{C}_{\mathrm{S}}$ on the left and $\mathrm{C}_{\mathrm{L}}$ on the right of the figures. $(a)$ The normalized critical Rayleigh number $R a_{c} / R a_{c 0}$, where $R a_{c 0} \approx 1706.7,(b)$ the critical wavenumber $k_{c},(c)$ the preferred orientation $\phi_{c}$, and (d) the frequency $\omega_{c}$.

figure $3(b)$ of Hoffman et al. (1998), albeit at a Taylor number that corresponds roughly to our $\tau=200$.

As the value of $\tau$ decreases, the shear layer loses its complicated spiral structure. Indeed the non-rotating plane Couette limit $\tau=0$ is well known to be stable to infinitesimal disturbances. It is therefore no surprise to find the type II and I stability boundaries $\left(\tau, R e_{c}\right)$, portrayed in figure 3 , rising steeply to infinity as $\tau$ decreases.

\subsection{Convective instability: $\operatorname{Pr}=1, R a \neq 0$}

We return now to the convection problem with rotation vertical $(\vartheta=0)$. In view of the large number of dimensionless parameters, we simplify our development by restricting attention to $\mathrm{Pr}=1$. In searching for neutral modes, we regard the Rayleigh number $R a$ and frequency $\omega$ as functions of the physical parameters $\tau$ and $R e$ as well as the mode parameters $k$ and $\phi$. At given $(\tau, R e)$ we minimize $R a$ over the $(k, \phi)$-plane to obtain the critical values $\left(R a_{c}, \omega_{c}, k_{c}, \phi_{c}\right)$.

We studied the nature of the solutions by varying $\tau$ at various fixed values of the Reynolds number $R e$. We found that the major physical interactions were clearly illustrated by the choice $R e=500$, to which we restrict our numerical description here. The corresponding critical values are plotted in figure 5. Four distinct types of mode are preferred in successive $\tau$-ranges, which we label $\mathrm{C}_{\mathrm{S}}, \mathrm{I}, \mathrm{II}, \mathrm{C}_{\mathrm{L}}$ corresponding to increasing values of $\tau$. Here I and II can be identified with Ekman instability modes. $\mathrm{C}$ refers to a principally convective instability; the labels $\mathrm{S}$ and $\mathrm{L}$ refer to smalland large- $\tau$ branches which may or may not be connected, depending on parameter 
(a)

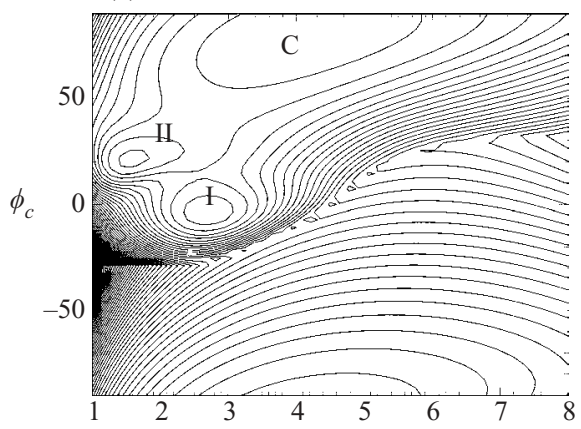

(c)

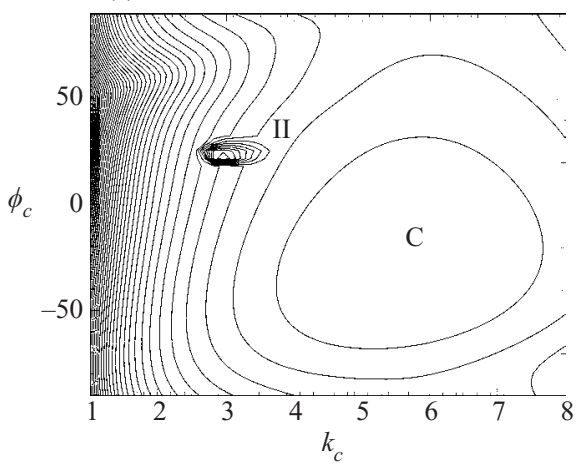

(b)

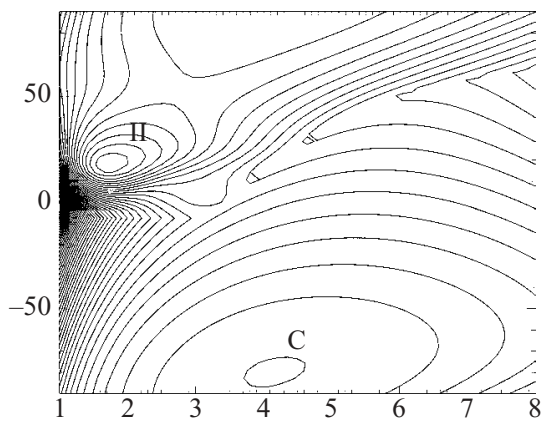

(d)

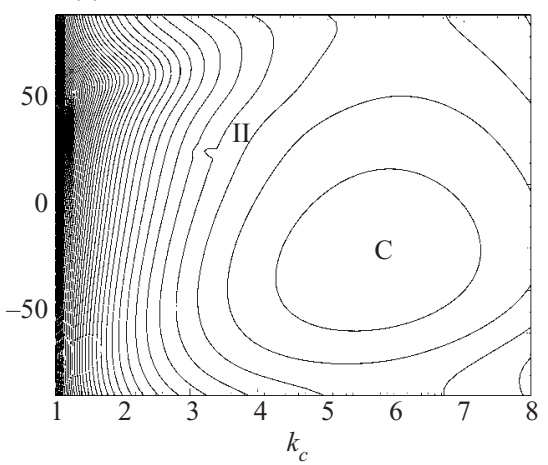

FiguRE 6. Contours of constant critical Rayleigh number $R a_{c}$ in the wave vector plane parameterized by $\left(k_{c}, \phi_{c}\right)$, for the case of vertical rotation $\vartheta=0, \operatorname{Pr}=1, R e=500$. Type I, II and $\mathrm{C}$ minima are labelled. Four different rotation rates are shown: $(a) \tau=40,(b) \tau=60$, (c) $\tau=160$, and $(d) \tau=170$.

values. To understand how these results emerge, we take four representative values of $\tau$ and plot contours of constant neutral $R a$ in the $(k, \phi)$-plane in figure 6. On each of them, we can isolate local minima which include modes I, II and C. Evidently, these local minima compete to be the global minimum identified in figure 5. Interestingly the convective minima $\mathrm{C}_{\mathrm{S}}$ and $\mathrm{C}_{\mathrm{L}}$ actually connect with each other as $\tau$ is increased from 0 to $\infty$ and so we can unambiguously label this the convective minimum $\mathrm{C}$ throughout. This agreeable state of affairs should be contrasted with the oblique rotation cases investigated in $\S 4.2$ below, for which the $C_{S}$ and $C_{L}$ branches are disconnected. Generally, there is a value of $\tau$ at which a local minimum evaporates. The corresponding points, which terminate the curves on figures 5, 11, 14 and 15 , are marked by diamonds.

To understand the nature of the various modes we examine the eigenfunctions, showing $v, \psi$ and perturbation temperature $\theta$ in figures 7, 8 and 9. Here we adopt the frame co-moving with the wave at its phase velocity $-\omega_{c} / k_{c}$, as in figure 4 .

\subsubsection{Small Taylor number $\tau^{2} \ll 1: R e=500$; type $C_{S}$}

When $\tau=0$, the roll axes are aligned, $\phi_{c}=0$, with the plane Couette shear $\lambda_{1}=0, \lambda_{2}=-1+z$. Consequently, the $\Psi, \Theta$-equations decouple from the $V$-equation within the system (2.21). As a result, neither the applied shear nor the induced $v$ interact with the convection, and so the critical Rayleigh number takes the value $R a_{c}=R a_{c 0}$ corresponding to the classical Rayleigh-Bénard problem mentioned in 


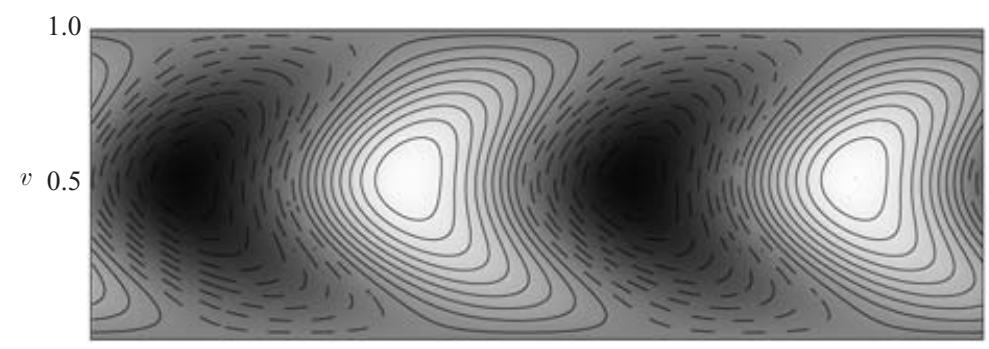

(a)
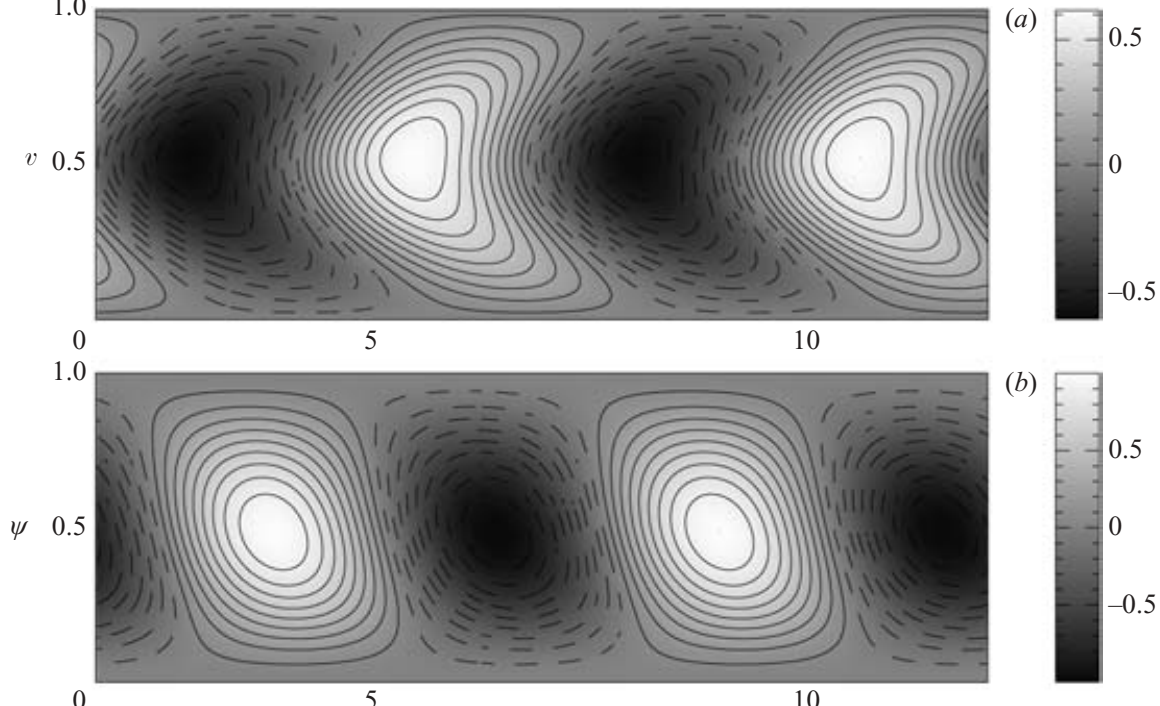

5

10
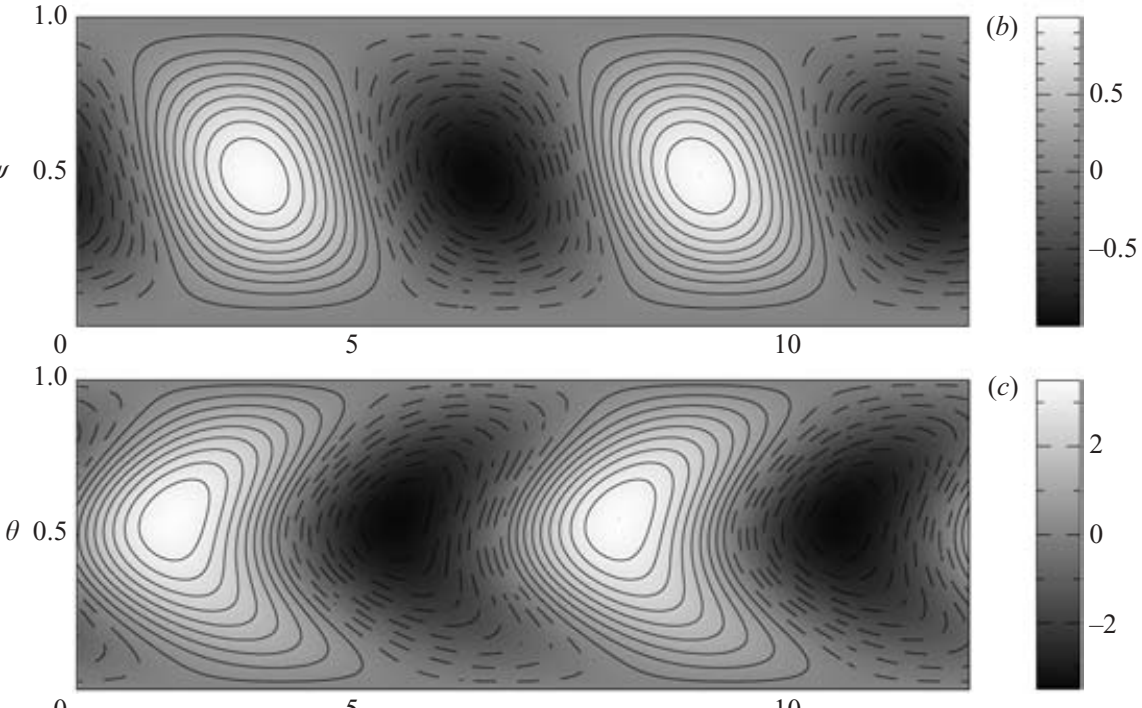

5

10

0

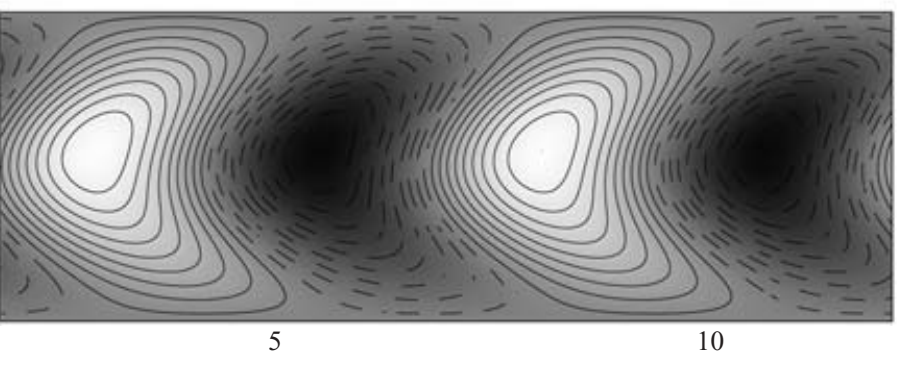

(c)

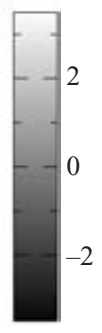

FIGURE 7. Contour plots describing the onset of convection in the co-moving $\left(x+\left(\omega_{c} / k_{c}\right) t, z\right)$-frame for the case $\vartheta=0, \operatorname{Pr}=1, \operatorname{Re}=500$. They illustrate a type $\mathrm{C}_{\mathrm{S}}$ mode at $\tau=2$, for which $R a_{c} \approx 4105.06, k_{c} \approx 3.271, \phi_{c} \approx 1.6^{\circ}$ and $\omega_{c} \approx 108.75$. (a) The toroidal velocity $v,(b)$ the stream function $\psi$, and $(c)$ the heat perturbation $\theta$.

the Introduction. Interestingly, with our choice $\operatorname{Pr}=1$ the equations governing $V$ and $\Theta$ are equivalent except for different constants multiplying the common source term $\mathrm{i} k \Psi$, and give $V=-\left(R e / R a_{c 0}\right) \Theta$.

For small Taylor number $\tau^{2} \ll 1$ the preferred mode $C_{S}$ identified in figure 5 is a perturbation of the $\tau=0$ solution. It is well known that rotation by itself is stabilizing. Nevertheless, the sharp rise of $R a_{c}$ versus $\tau$ suggests that the further interaction with the shear strengthens that stabilization. Indeed, the situation in the limit $R e \gg 1$ is quite clear. Though the shear is only weakly modified by the rotation,

$$
\lambda_{1} \sim(1-z)\left[\phi-\frac{1}{3} \mu^{2} z(2-z)\right], \quad \lambda_{2} \sim-1+z \text { for }|\phi| \ll 1,
$$

this is the most important effect because of the large value of the Reynolds number. Thus we can neglect rotation except where it modifies $\boldsymbol{\Lambda}$. Accordingly we need only consider the first and last components of (2.21) for $\Psi$ and $\Theta$, which involve the shear via $\lambda_{1}$ above; here the contribution to the convection made by $V$ is small, because of the relatively small size of $\tau$, and can be ignored. The shear associated with $\lambda_{1}^{\prime}$ tilts the isotherms, shortens the vertical length scale and enhances dissipation. The preferred mode minimizes that shear. For $U_{0}>0(<0)$, the rolls drift southward (northward) 

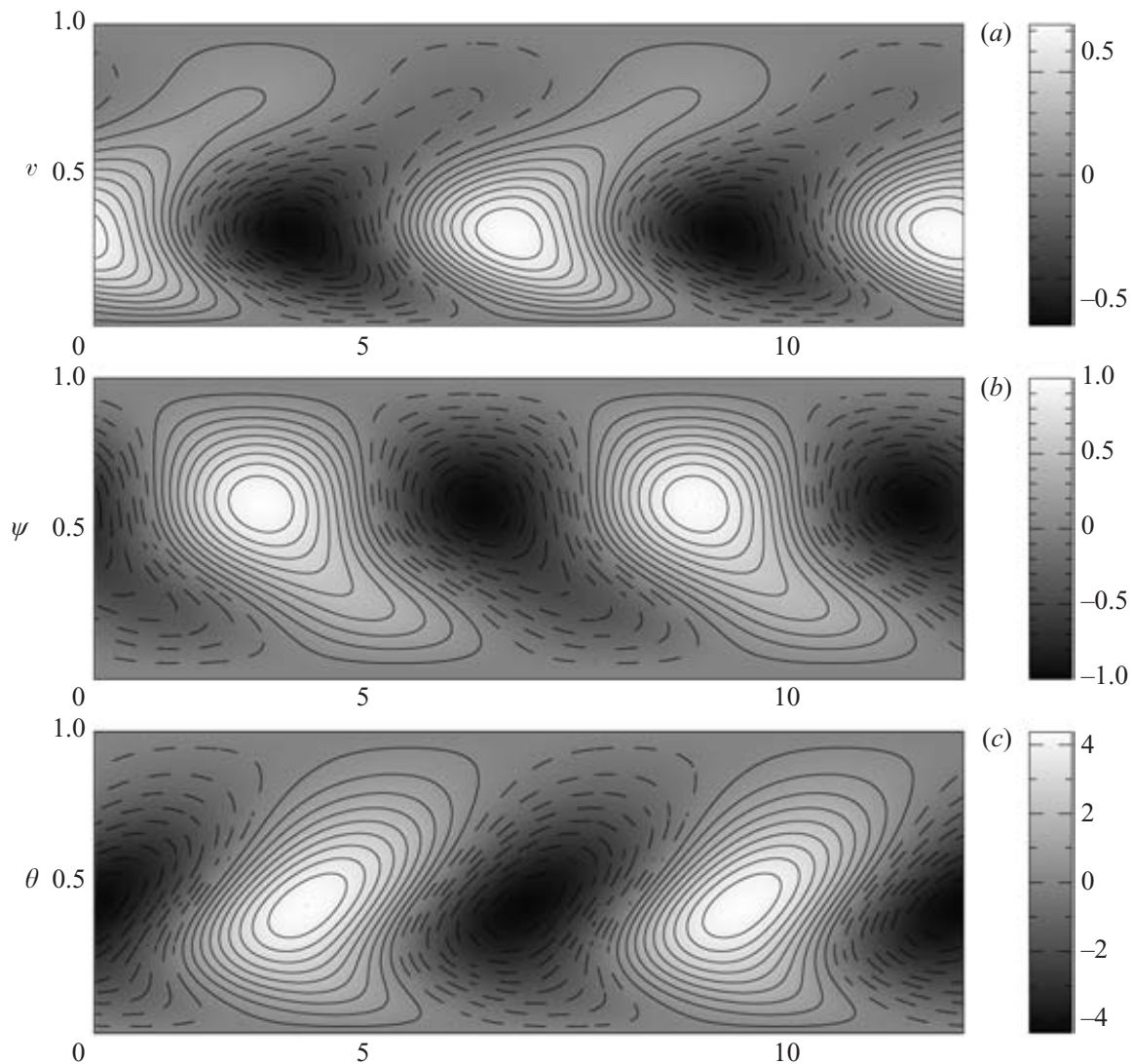

10

(c)

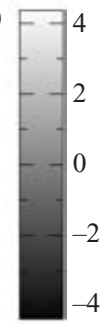

FIGURE 8. As in figure 7, except that now it illustrates a type I mode at $\tau=40$, for which $R a_{c} \approx 10064.92, k_{c} \approx 2.7079, \phi_{c} \approx-3.747^{\circ}$ and $\omega_{c} \approx 258.673$.

with a small phase velocity $-\omega_{c} / k_{c}$ in the direction of the mean flow $\int_{0}^{1} \Lambda_{2} \mathrm{~d} z$ and $0<\phi=O(\tau)$.

Though the case $\tau=2$, portrayed in figures 7 , is not for particularly small $\tau$ the features just mentioned are readily confirmed. The contours of constant $v$ and $\theta$ shown in figure $7(a, c)$ appear to be almost coincident. The value of $\phi$ selected suggests that $\lambda_{1}^{\prime}=0$ at roughly $z=1 / 2$. (Remember that vorticity is convected and not the stream function $\psi$ and so figure $7(b)$ does not give a direct indication of the shear.) The small roll tilt $\phi$ and the southward (northward) roll drift for $\operatorname{Re}>0(<0)$ are also clearly identified from the data in figure $5(b, c, d)$.

\subsubsection{Large Taylor number $\tau^{2} \gg 1$ : type $C_{L}$}

As $\tau \rightarrow \infty$ the Ekman layer width $O\left(\tau^{-1 / 2}\right)$ tends to zero and has virtually no influence on the convection. Therefore we may apply the well-known results applicable when there is no shear, $R e=0$. Consequently, the onset of convection is steady, the critical wavenumber is $k_{c}=(\pi \tau / \sqrt{2})^{1 / 3}$ and the corresponding critical Rayleigh number is $R a_{c}=3\left(\pi^{2} / 2\right)^{2 / 3} \tau^{4 / 3}$ (see (3.8) below). In the absence of shear, however, the system is invariant to rotations about the $z$-axis and so no roll orientation is determined. In the Appendix we develop an asymptotic expansion for large $\tau$, which is applicable over a wide range of $R e$. The analysis of $\S 3.3$ below shows that the small Ekman suction into the Ekman layer controls the orientation of the convection 


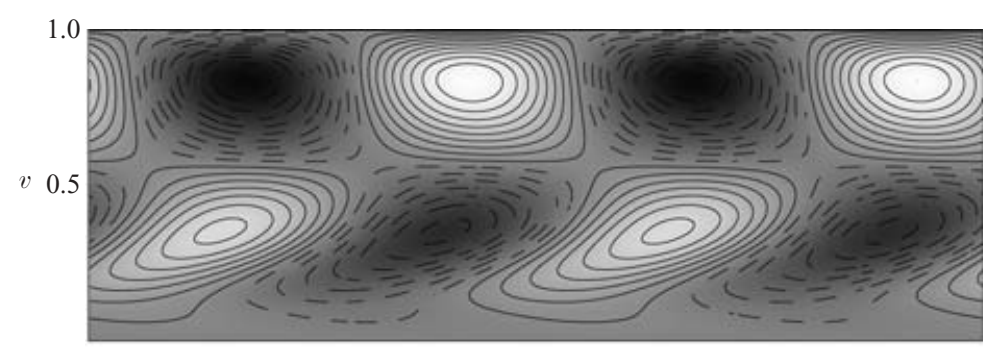

(a)
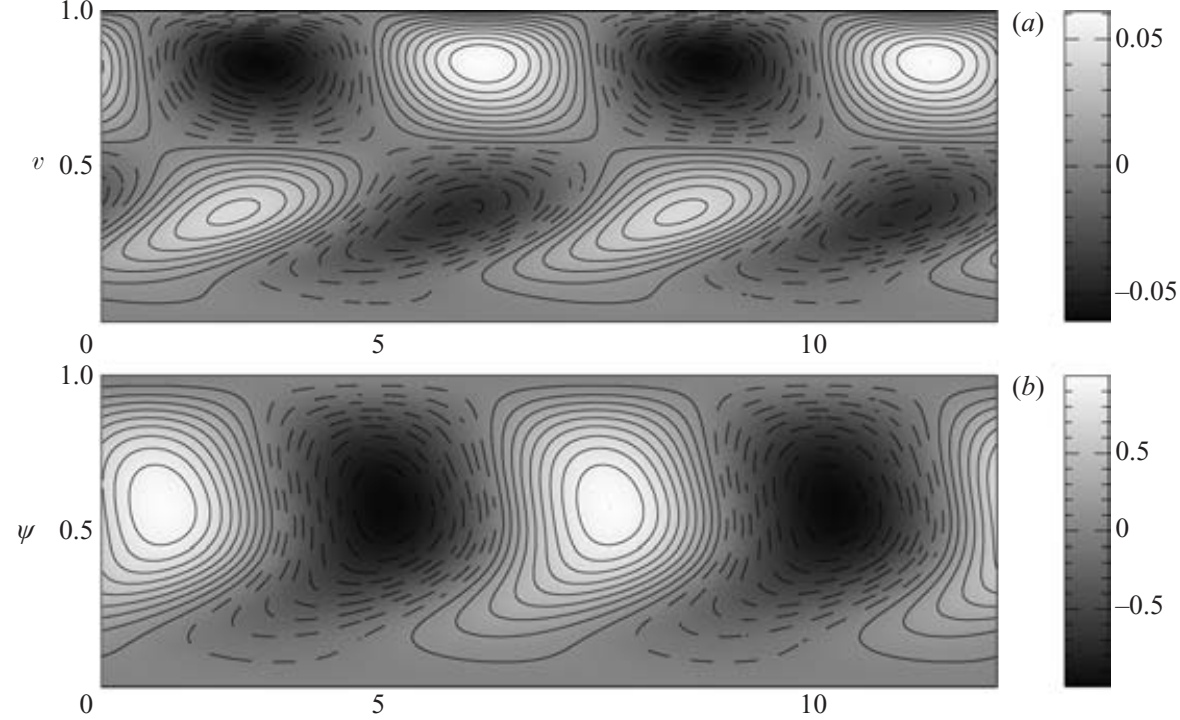

5

10

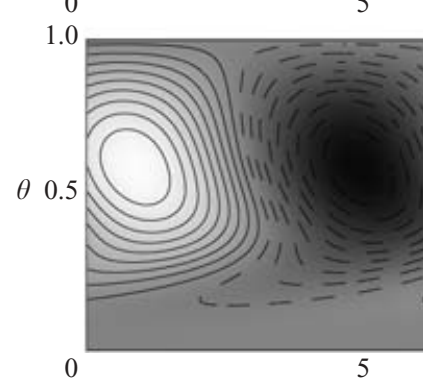

10

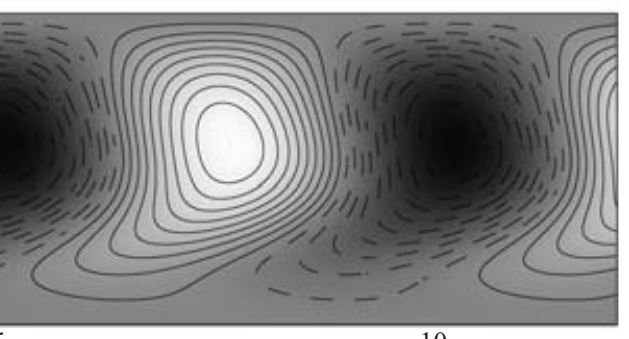

(b)

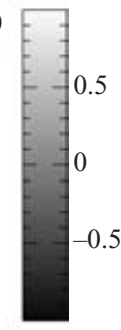

(c)

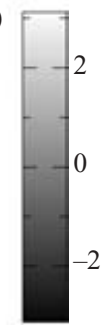

FIGURE 9. As in figure 7, except that now it illustrates a type $\mathrm{C}_{\mathrm{L}}$ mode at $\tau=200$, for which $R a_{c} \approx 13652.27, k_{c} \approx 6.0025, \phi_{c} \approx 154.44^{\circ}$ and $\omega_{c} \approx 13.73$.

rolls, which is found to take the value $\phi_{c}=-27.0451^{\circ}$ (or equivalently $152.9548^{\circ}$ ). The corresponding critical frequency is given by (3.11) below.

The large- $\tau$ solution retains its character down to moderately large $\tau$, where it is identified in figure 5 as a mode $C_{L}$. Indeed, the critical values $R a_{c}$ and $k_{c}$ agree well with the asymptotic values; the convection is almost stationary and $\phi_{c}$ has achieved the value of $152.95^{\circ}$. The mode's convection characteristics illustrated in figure 9 certainly support that point of view. It is also clear that the convection takes place largely outside the moderately thin Ekman layer. Indeed any overshooting into the Ekman layer is rapidly sheared and distorted.

\subsubsection{Moderate Taylor numbers: $R e=500$; types $I$ and $I I$}

From figure 5 we notice that there are two values of $\tau$, roughly 60 and 160, at which $R a_{c}$ vanishes. These two values are determined by the intersection of the line $R e_{c}=500$ with the (solid) type II curve on figure 3. This means that the mode identified on figure 5 is essentially a hydrodynamic instability of type II modified by the presence of buoyancy forces. Indeed, in the negative- $R a_{c}$ range, where the type II disturbance is hydrodynamically unstable, the mode is only stabilized by the application of a stable stratification. In view of this hydrodynamic identification it is natural to continue to label it as type II even when the buoyancy force is involved. 
By examining the stability characteristics of the mode labelled I on figure 5, it is clearly a modification of the type I hydrodynamic mode; compare $k_{c}, \phi_{c}$, $\omega_{c}$ with those shown on figure $3(b, c, d)$. Unlike the type II mode, which exhibits instability at $R a=0$, this does not because at $R e=500$ the type I mode is hydrodynamically stable (see figure $3 a$ ). We exhibit an example of this type I mode in figure 8 for $\tau=40$, where the minimum values of $R a$ for the type I and II modes coincide. The dynamical features of this type I mode, particularly the concentration of the $v$-velocity inside the shear layer, are very similar to the type II mode, which occurs at almost the same Rayleigh number. Indeed, this type I mode even bears a striking resemblance to the type II hydrodynamic mode illustrated in figure 4. One feature that is evident from figure 8 is that $v$ is generated high in the Ekman layer. The overall picture suggests that energy is being tapped primarily from the shear but the buoyancy force is also playing an essential role in driving the motion.

Just as in the case of the small- $\tau$ convective $C_{S}$ modes, $\phi_{c}$ is small for these type I modes, suggesting that the roll axis is adjusting its orientation to prevent shearing by the underlying Ekman layer flow from having too strong a stabilizing effect. Despite this tuning, considerable shearing is evident in figure 8. Furthermore, since $\phi_{c}$ is small, these type I convective modes have roll axes which are aligned almost east-west and travel southwards (northwards) for $U_{0}>0(<0)$.

The identification of the global minimum is a numerically delicate matter and that is why we have produced the contour plots of constant neutral Rayleigh number $R a$ in figure 6. In interpreting these plots it important to appreciate that they are $\pi$ (or $180^{\circ}$ ) periodic in the $\phi$-direction, i.e. the top boundary of each contour plot is identical to its bottom boundary. We find that up to three local minima can be identified at each value of $\tau$. For the case $\tau=40$ illustrated in figure $6(a)$ the minima for type I and II modes have almost identical $R a$, while the mode $\mathrm{C}_{\mathrm{L}}$ minimum is greater. As $\tau$ increases the type I minimum evaporates as illustrated in figure $6(b)$ for the value $\tau=60$, shortly after the type II hydrodynamic instability sets in. With further increase in $\tau$ the type II mode minimum sharpens as illustrated in figure $6(c)$ for the value $\tau=160$ at which the type II hydrodynamic instability is cut off. Indeed with further increase of $\tau$ the type II mode minimum becomes increasingly difficult to track. By $\tau=170$, illustrated in figure $6(d)$, though the local minimum of the type II mode continues to exist, it is very tiny. With further increases of $\tau$ it soon evaporates and we are left with the global minimum associated with the large- $\tau$ convective $C_{L}$ mode.

\subsection{Large rotation rate $\tau \gg 1, \tau^{2 / 3} \gg R e$}

In this subsection (and the Appendix) we sketch the limit of rapid rotation with vertical rotation vector $\vartheta=0$. For non-zero Reynolds number $R e$, the solution splits into two parts. One part is the mainstream solution valid outside the Ekman layer, where the applied shear is negligible. The other is the boundary layer solution valid inside the Ekman layer itself. The structure of this boundary layer determines the orientation of rolls at onset.

With $\left(\lambda_{1}, \lambda_{2}\right)=\mathbf{0}$ in the main stream, the governing equations (2.21) have a solution of the form

$$
\Psi=\bar{\Psi} \sin l(z-\bar{z}), \quad V=\bar{V} \cos l(z-\bar{z}), \quad \Theta=\bar{\Theta} \sin l(z-\bar{z}),
$$

where $\bar{\Psi}, \bar{V}, \bar{\Theta}, l$ and $\bar{z}$ are complex constants, provided that

$$
\frac{k^{2} R a}{k^{2}+l^{2}+\mathrm{i} \omega}=\left(k^{2}+l^{2}+\mathrm{i} \operatorname{Pr}^{-1} \omega\right)\left(k^{2}+l^{2}\right)+\frac{l^{2} \tau^{2}}{k^{2}+l^{2}+\mathrm{i} \operatorname{Pr}^{-1} \omega} .
$$


We now assume that the Ekman jump conditions take the form

$$
\Psi\left(1_{-}\right)=-\tau^{-1 / 2} \Gamma_{0} V\left(1_{-}\right), \quad \Psi\left(0_{+}\right)=\tau^{-1 / 2} \Gamma_{B} V\left(0_{+}\right),
$$

where $\Gamma_{0}$ and $\Gamma_{B}$ are $O(1)$ complex parameters determined by the boundary layer solution. On the further assumption that $k=O\left(\tau^{1 / 3}\right)$, we see that $\bar{V} \sim\left(\tau l / k^{2}\right) \bar{\Psi}$ and then the boundary conditions (3.5) are met by (3.3), when

$$
\bar{z} \sim 1+\tau^{1 / 2}\left(\Gamma_{0} / k^{2}\right), \quad l \sim \pi\left[-1+\tau^{1 / 2}\left(\Gamma / k^{2}\right)\right] \quad\left(\Gamma=\Gamma_{0}+\Gamma_{B}\right),
$$

where the Ekman layer contributions are small, $O\left(\tau^{-1 / 6}\right)$. Correct to leading order the real and imaginary parts of (3.4) now yield

$$
\begin{aligned}
R a \sim & \frac{\tau^{2}}{k^{2}}\left[\pi^{2}+\frac{k^{6}}{\tau^{2}}-\frac{2 \pi^{2} \tau^{1 / 2}}{k^{2}} \operatorname{Re}\{\Gamma\}+O\left(\tau^{1 / 3}\right)\right], \\
& \operatorname{Pr}^{-1} \omega \sim \frac{2 \pi^{2} \tau^{1 / 2}\left[\operatorname{Im}\{\Gamma\}+O\left(\tau^{-1 / 6}\right)\right]}{(P r-1) \pi^{2}+(P r+1) k^{6} / \tau^{2}} .
\end{aligned}
$$

From (3.7a), we recover the critical Rayleigh number and wavenumber for rapidly rotating convection:

$$
R a_{c} \sim 3\left(\pi^{2} / 2\right)^{2 / 3} \tau^{4 / 3}, \quad k_{c} \sim(\pi \tau / \sqrt{2})^{1 / 3} .
$$

With $(3.7 b)$ they determine the critical frequency:

$$
\operatorname{Pr}^{-1} \omega_{c} \sim \frac{4 \tau^{1 / 2} \operatorname{Im}\{\Gamma\}}{3 \operatorname{Pr}-1} \quad\left(\operatorname{Pr} \neq \frac{1}{3}\right) .
$$

To complete the solution, we require the value of $\Gamma$ as determined by the Ekman layer solution. The appropriate boundary layer problem is formulated in the Appendix in terms of the parameter $\Delta=\mathrm{i} k \operatorname{Re} / \tau$ (see $(\mathrm{A} 3 b)$ ), on the basis that $\operatorname{Re}=O\left(\tau^{2 / 3}\right)$ (equivalently $|\Delta|=O(1)$ ). Essentially, in that limit, we may ignore buoyancy forces in the Ekman layer and solve the equations governing $\Psi$ and $V$ alone. In the limit

$$
R e \ll \tau^{2 / 3}, \quad \text { namely }|\Delta| \ll 1,
$$

a series solution is constructed in the Appendix, which determines the real and imaginary parts of $\Gamma_{B}$. We also have the standard Ekman layer suction result $\Gamma_{0}=$ $1 / \sqrt{2}$ (see e.g. Greenspan 1968). From $(3.7 a)$ it is clear that the critical value of $\phi_{c}$ is obtained by maximizing $\operatorname{Re}\left\{\Gamma_{B}\right\}$ with respect to $\phi$. The dependence on $\phi$ occurs at $O\left(|\Delta|^{2}\right)$ and maximization determines $\phi_{c} \approx-27.0451^{\circ}$ (see (A 16)). At this angle the imaginary part of $\Gamma_{B}$ (see (A 17)) determines via (3.9) the critical frequency

$\operatorname{Pr}^{-1} \omega_{c} \sim \frac{4 \tau^{-1 / 2}}{3 \operatorname{Pr}-1}\left(\frac{\pi}{\sqrt{2}}\right)^{1 / 3} \Gamma_{1}\left(\phi_{c}\right)\left(\frac{R e}{\tau^{2 / 3}}\right), \quad$ where $\quad \Gamma_{1}\left(\phi_{c}\right) \approx-0.268652$.

As a check on the power law behaviour for large $\tau$, figure 10 displays numerical results for two cases and the asymptotic prediction. The solid lines are for the computation for fixed Reynolds number $R e=100$, for which $|\Delta| \rightarrow 0$ as $\tau \rightarrow \infty$. The dotted lines correspond to the variable Reynolds number $R e=\tau^{2 / 3}$, for which $|\Delta|=O(1)$ as $\tau \rightarrow \infty$. The dashed lines correspond to the asymptotic analytic values. Certainly $R a_{c}$ and $k_{c}$ shown on figure $10(a, b)$ approach the asymptotic forms (3.8). Somewhat surprisingly both cases illustrated in figure $10(c)$ appear to be approaching $\phi=152.95^{\circ} \equiv \phi_{c}+180^{\circ}$ as predicted by the small- $|\Delta|$ theory, which may suggest that the critical roll angle $\phi_{c}$ is not sensitive to the value of $|\Delta|$ (at least when it is $O(1))$. There is, however, some discrepancy with the critical frequency $\omega_{c}$ portrayed in 

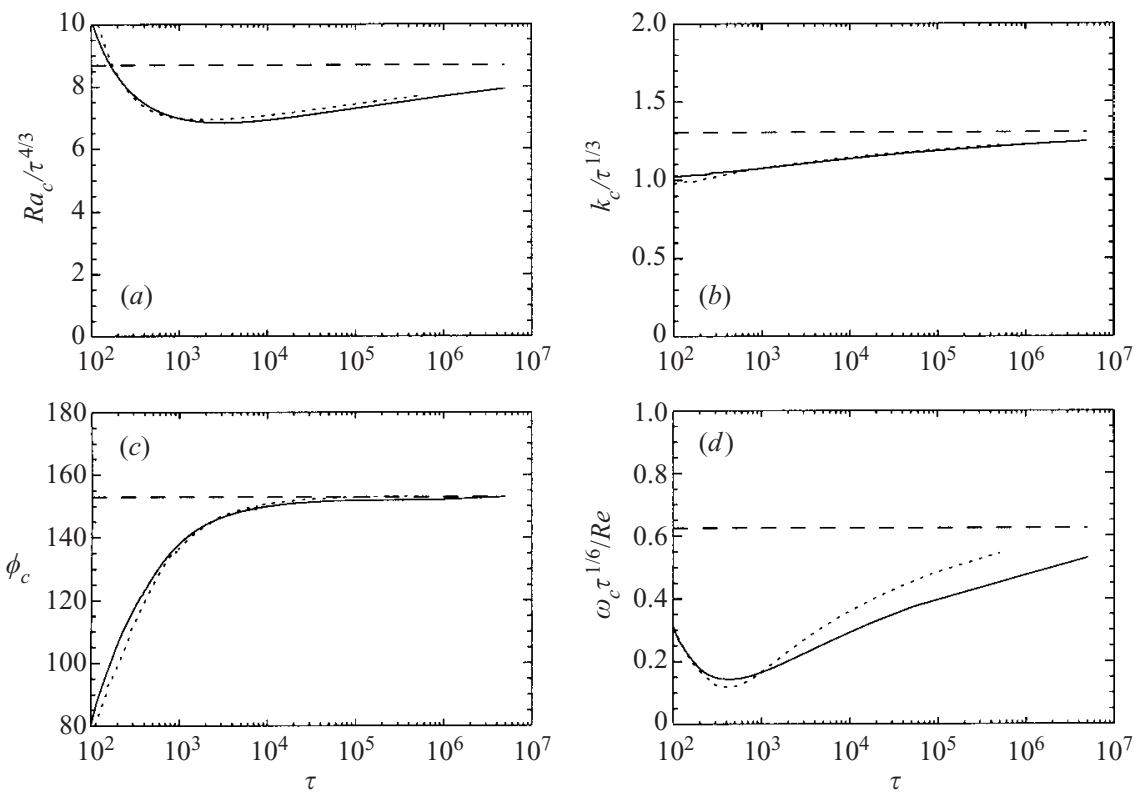

Figure 10. The critical values for vertical rotation $\vartheta=0, \operatorname{Pr}=1$, plotted against $\tau$ on a logarithmic scale. The cases illustrated are $R e=100$ (solid), $R e=\tau^{2 / 3}$ (dotted) and the asymptotic critical values (dashed). (a) The normalized critical Rayleigh number $R a_{c} / \tau^{4 / 3}$, (b) the normalized critical wavenumber $k_{c} / \tau^{1 / 3},(c)$ the preferred orientation $\phi_{c}$, and $(d)$ the normalized frequency $\omega_{c} \tau^{1 / 6} / R e$.

figure $10(d)$, but at any rate the correct power law is evident, which is the most that is to be expected. Note that the $180^{\circ}$ rotation of $\phi_{c}$ between the asymptotic theory and the numerical results leads to a sign change in $\omega_{c}$. It is associated with the symmetry $\phi \leftrightarrow \phi+180^{\circ}$ and $\omega \leftrightarrow-\omega$ at fixed $k$; the numerical results illustrate a positive $\omega_{c}$, whereas the asymptotic theory (3.11) determines a negative $\omega_{c}$.

\section{Oblique rotation $0<\vartheta<\pi / 2$}

In the previous section we discussed the case of vertical rotation $(\vartheta=0)$, while we explained in the Introduction that Kropp \& Busse (1991) have examined the case of horizontal rotation $(\vartheta=\pi / 2)$. Here we consider the general case of oblique rotation $(0<\vartheta<\pi / 2)$. In $\S 4.1$ we consider only hydrodynamic instability without buoyancy forces $(R a=0)$. In $\S 4.2$ a numerical study of buoyancy-related instabilities $(R a \neq 0)$ is undertaken which largely focuses on the representative case $\vartheta=\pi / 4$. In $\S 4.3$ an asymptotic theory is developed for rapid vertical rotation $|\tau \cos \vartheta| \gg 1$, which like $\S 3.3$ builds on the analysis of suction in the Ekman boundary layer given in the Appendix.

We emphasize throughout that the character of the Ekman-Couette shear layer for $\vartheta \neq 0$ depends only on the vertical component of rotation $\boldsymbol{\Omega}_{v}$, whereas the stability characteristics of the system are sensitive to the sign of the horizontal component or more precisely the triple product $\mathscr{T} \equiv \boldsymbol{U}_{0} \cdot(\boldsymbol{g} \times \boldsymbol{\Omega})$, introduced below (2.14), which highlights the sign of $U_{0}$ defining the direction of motion of the lower boundary. Quite simply the $U_{0} \leftrightarrow-U_{0}$ symmetry appealed to in $\S 3$ in interpreting results is now broken by the horizontal component of rotation $\boldsymbol{\Omega}_{h}(\neq \mathbf{0})$ and is no longer applicable. 

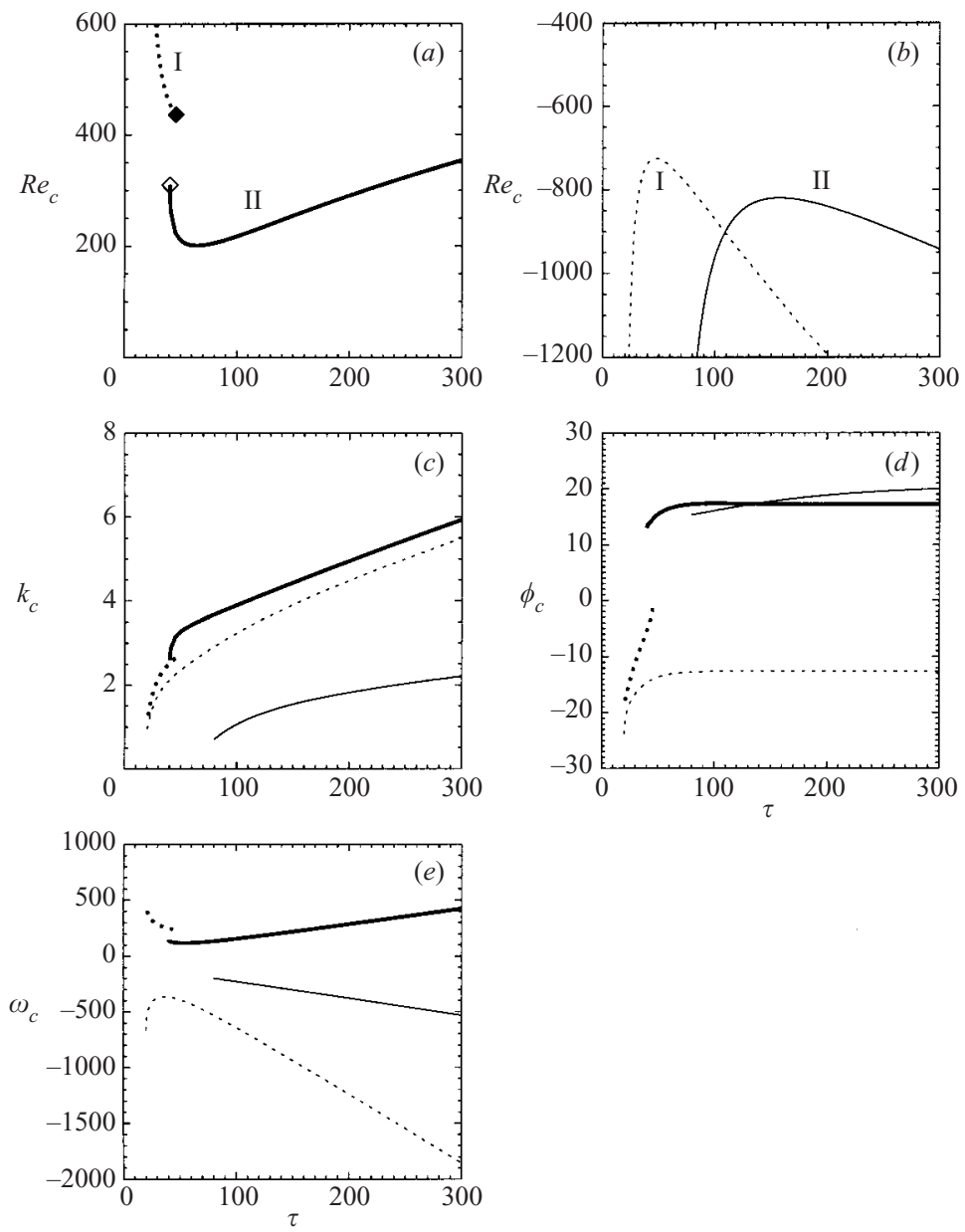

FIGURE 11. As in figure 3 but now for the case $\vartheta=\pi / 4$, except that positive and negative $R e$ are now shown on separate figures $(a)$ and $(b)$ respectively. Positive (negative) type I modes are distinguished by thick (thin) dotted curves, while type II modes are distinguished by thick (thin) solid curves. The disappearance of the type I and type II local minima are located approximately by the solid and hollow diamonds respectively.

\subsection{Ekman layer instability $R a=0$}

Let us briefly remark on the limiting plane Couette case $\left(\left|\boldsymbol{\Omega}_{v}\right|=0\right)$ at $\vartheta=\pi / 2$. The mathematical formulation of the onset of instability is identical to the classical Rayleigh-Bénard problem. From that it is easy to deduce that instability is possible provided that $R e>2 \sqrt{ } R a_{c 0}(>0)$ and then in the Taylor number range $(0<) \tau_{-}<$ $\tau<\tau_{+}$, where $\tau_{ \pm}=\left[R e \pm \sqrt{ }\left(R e^{2}-4 R a_{c 0}\right)\right] / 2$. This result illustrates the important fact that the cases of positive and negative $U_{0}$ are not equivalent, when the rotation has a horizontal component $\left(\left|\boldsymbol{\Omega}_{h}\right| \neq 0\right)$.

When there is a vertical component of rotation $\left(\left|\boldsymbol{\Omega}_{v}\right| \neq 0\right.$ for $\left.\vartheta \neq 0\right)$, the flow is of Ekman-Couette type which becomes an Ekman layer in the limit $\vartheta$ fixed, $\tau \rightarrow \infty$. In figure 11 we illustrate the characteristics of the critical modes as functions of $\tau$ for the representative inclination $\vartheta=\pi / 4$ of the rotation. The stability curves are plotted for both signs of the Reynolds number corresponding to the two opposite signs of 

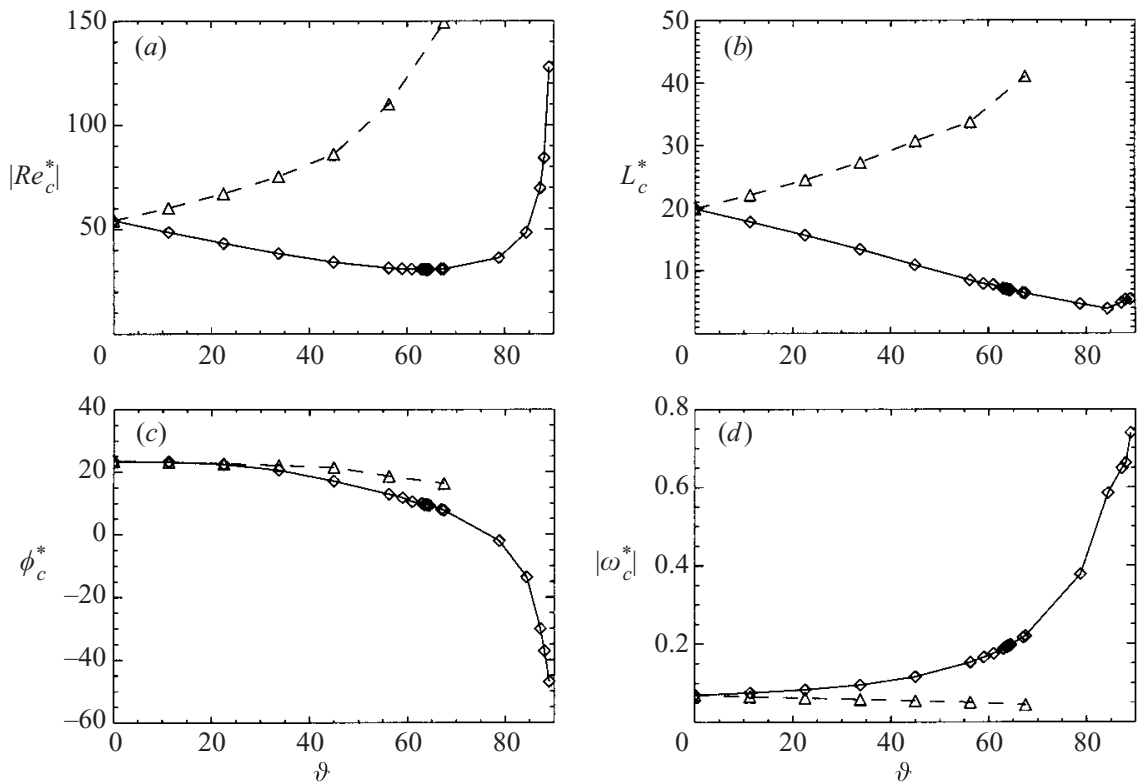

FiguRE 12. Critical values for the Ekman layer instability plotted against the co-latitude $\vartheta$ of the oblique rotation. The case $\operatorname{Re}>0(<0)$ with $\omega>0(<0)$ is shown as a solid curve and diamond markers (dashed curve and triangle markers). (a) The magnitude of the critical Reynolds number based on the Ekman layer depth, namely $\left|R e_{c}^{*}\right|,(b)$ the critical wavelength of the instability in units of the Ekman layer depth $D$, namely $L_{c}^{*},(c)$ the critical preferred angle $\phi_{c}^{*}$, and $(d)$ the normalized frequency $\left|\omega_{c}^{*}\right|$.

the moving bottom velocity $U_{0}$; instability occurs when $\pm R e> \pm R e_{c}(>0)$. Note that the two branches for both positive and negative $R e_{c}$ correspond to the type I and II branches illustrated in figure 3. In fact what has happened is that the positive and negative $R e$ stability characteristics are identical for $\vartheta=0$, namely $\left(R e_{c}, k_{c}, \phi_{c}, \omega_{c}\right) \leftrightarrow$ $\left(-R e_{c}, k_{c}, \phi_{c},-\omega_{c}\right)$. As $\vartheta$ increases from zero, an asymmetry develops which is typified by our illustrative case $\vartheta=\pi / 4$. Significantly, in view of our remarks about the plane Couette flow case, the positive-Re modes become unstable for smaller $\left|U_{0}\right|$ than for negative $R e$.

As $|\tau \cos \vartheta| \rightarrow \infty$ the Ekman layer adjacent to the lower boundary thins indefinitely and a well-defined Ekman instability is identified as discussed previously by Leibovich $\&$ Lele (1985). In this limit the asymptotic behaviours of the curves in figure $11(a-d)$ have the functional forms

$$
\left.\begin{array}{ll}
R e_{c}=\operatorname{Re}_{c}^{*}(\vartheta) \mu, & k_{c}=2 \pi \mu / L_{c}^{*}(\vartheta), \\
\phi_{c}=\phi_{c}^{*}(\vartheta), & \omega_{c}=\operatorname{Re} \operatorname{Pr} \mu \omega_{c}^{*}(\vartheta)
\end{array}\right\} \quad \text { on } \quad 0 \leqslant \vartheta<\pi / 2,
$$

where as usual $\mu=\sqrt{\tau(\cos \vartheta) / 2}$. Here the scaled Reynolds number $R e_{c}^{*}$, the wavelength $L_{c}^{*}$ and the frequency $\omega_{c}^{*}$ are based, as in $\S 3.1$, on units of the Ekman layer depth $D=\sqrt{v /(\Omega \cos \vartheta)}$, which is now a function of co-latitude $\vartheta$, and the time scale $D / U_{0}$. This latitudinal scaling of the critical quantities is adopted for consistency with the units adopted by Leibovich \& Lele (1985).

Plots of the critical values $R e_{c}^{*}, L_{c}^{*}, \phi_{c}^{*}, \omega_{c}^{*}$ on $0 \leqslant \vartheta<\pi / 2$ are shown on figure 12: all critical values correspond to type II modes. The data points at each fixed $\vartheta$ were obtained in the following way. First, data for the critical values $R e_{c}, L_{c}, \phi_{c}, \omega_{c}$ were 
obtained for various $\tau$ at each $\vartheta$. Secondly, for sufficiently large $\tau$ these data were fitted to the asymptotic formulas (4.1) so as to extract the quantities $R e_{c}^{*}, L_{c}^{*}, \phi_{c}^{*}, \omega_{c}^{*}$ relevant to the Ekman instability in a semi-infinite region of fluid. At $\vartheta=0$, we have the type II minimum $R e_{c}^{*} \simeq 54.21$ as noted in $\S 3.1$. On increasing $\vartheta$, while keeping the magnitude $\left|\boldsymbol{\Omega}_{v}\right|$ of the vertical rotation fixed (i.e. constant $\mu$ ), it is clear from figure 12(a) that the effect of increasing the horizontal rotation $\left|\boldsymbol{\Omega}_{h}\right|$ is to lower (raise) the magnitude $\left|R e_{c}^{*}\right|$ of the critical Reynolds number, when $R e_{c}^{*}>0\left(R e_{c}^{*}<0\right)$. This destabilization (stabilization) of the Ekman layer flow is consistent with our results for the case $\vartheta=\pi / 4$ portrayed in figure 11(a) (11(b)) at finite $\tau$.

We find that $\operatorname{Re}_{c}^{*}(\vartheta)$ is minimized on figure $12(a)$ at $\vartheta=\vartheta_{\text {min }}$, where the corresponding critical values are

$$
\left.\begin{array}{ll}
R e_{c}^{*}\left(\vartheta_{\min }\right) \approx 30.8246, & L_{c}^{*}\left(\vartheta_{\min }\right) \approx 6.9741, \\
\phi_{c}^{*}\left(\vartheta_{\min }\right) \approx 9.53^{\circ}, & \omega_{c}^{*}\left(\vartheta_{\min }\right) \approx 0.1943
\end{array}\right\} \quad \text { with } \quad \vartheta_{\min } \approx 64.02^{\circ}
$$

Leibovich \& Lele (1985) considered a more general problem in which the shear velocity has arbitrary orientation $\boldsymbol{U}_{0}=U_{0} \cos \alpha \widehat{\boldsymbol{X}}+U_{0} \sin \alpha \widehat{\boldsymbol{Y}}$ outside the boundary layer and vanishes on the boundary itself. Our studies with $R e>0$ and $R e<0$ correspond to the special cases $\alpha=180^{\circ}$ and $\alpha=0^{\circ}$ respectively. Though the results listed in their table 1 follow a minimization over $\alpha$, the data of their bottom row apply to all latitudes less than $26.2^{\circ}$. On the co-latitude range $63.8^{\circ}<\vartheta<90^{\circ}$, their results correspond to the orientation $\alpha=180^{\circ}$ at $\vartheta=\tan ^{-1}\left[2.033 / \cos \left(9^{\circ}\right)\right] \approx 64.08^{\circ}$. In turn these values determine $R e_{c}^{*}=30.8, L_{c}^{*}=2 \pi / 0.89 \approx 7.06, \varphi_{c}^{*}=9^{\circ}$ and $\omega_{c}^{*}=0.89 \times\left(0.375-\sin 9^{\circ}\right) \approx 0.1945$, where we have noted that their phase velocity must be adjusted to accommodate the fact that their boundary is stationary whereas ours moves with velocity $\boldsymbol{U}_{0}$. These results agree nicely with our results (4.2) obtained by minimizing over $\vartheta$ at $\alpha=0^{\circ}$.

For strictly horizontal rotation $\vartheta=\pi / 2$, the critical Reynolds number follows the Taylor vortex scaling, $R e_{c}=O(\tau)$. For nearly horizontal rotation $\vartheta \simeq \pi / 2$, there is a transition from this scaling to the Ekman type II scaling. More precisely the plane Couette-Taylor vortex limit is applicable when $\mu \ll 1$ (i.e. $\tau \ll(\cos \vartheta)^{-1}$ ) and the Ekman layer limit $R e_{c}=O\left(\tau^{1 / 2}\right)$ is applicable when $\mu \gg 1$ (i.e. $\left.\tau \gg(\cos \vartheta)^{-1}\right)$. We illustrate the transition between the two limiting cases by the thick continuous curve on figure 13 for the case $\vartheta=89.5^{\circ}$. The picture is complicated by a new mode, which emerges at $\tau \approx 217.5$ (i.e. $\mu \approx 0.974$ ). It too follows the Taylor vortex scaling at moderate $\mu$. It then behaves in a very different way, tending to line up its roll axis with the large horizontal component of rotation $\boldsymbol{\Omega}_{h}$, i.e. $\phi_{c} \rightarrow \pi / 2$ as $\mu \rightarrow \infty$. This alignment attempts to minimize the inhibiting effect of rotation and leads to a low frequency in much the same way as the large- $\tau$ convective $C_{L}$ modes do, which we discuss in $\S 4.3$ below. Otherwise the mode appears simply to be yet another Ekman layer instability, which becomes important at low latitudes. A mode with very similar properties was identified by Hoffmann \& Busse (1999) at moderate $\tau$ and for a significant $\vartheta$ range between roughly $40^{\circ}$ and $90^{\circ}$. They called it a Taylor vortex mode because theirs linked directly with the Taylor vortex solution at $\vartheta=90^{\circ}$. Of course, our results do not show a direct link and that is why we prefer to call this a low-latitude Ekman layer mode. It is important to remember that the Hoffmann \& Busse (1999) model is different to ours in that their upper and lower boundaries move with equal magnitudes in opposite directions. The fact that their low-latitude Ekman layer mode links to the Taylor vortices and ours does not may simply be 

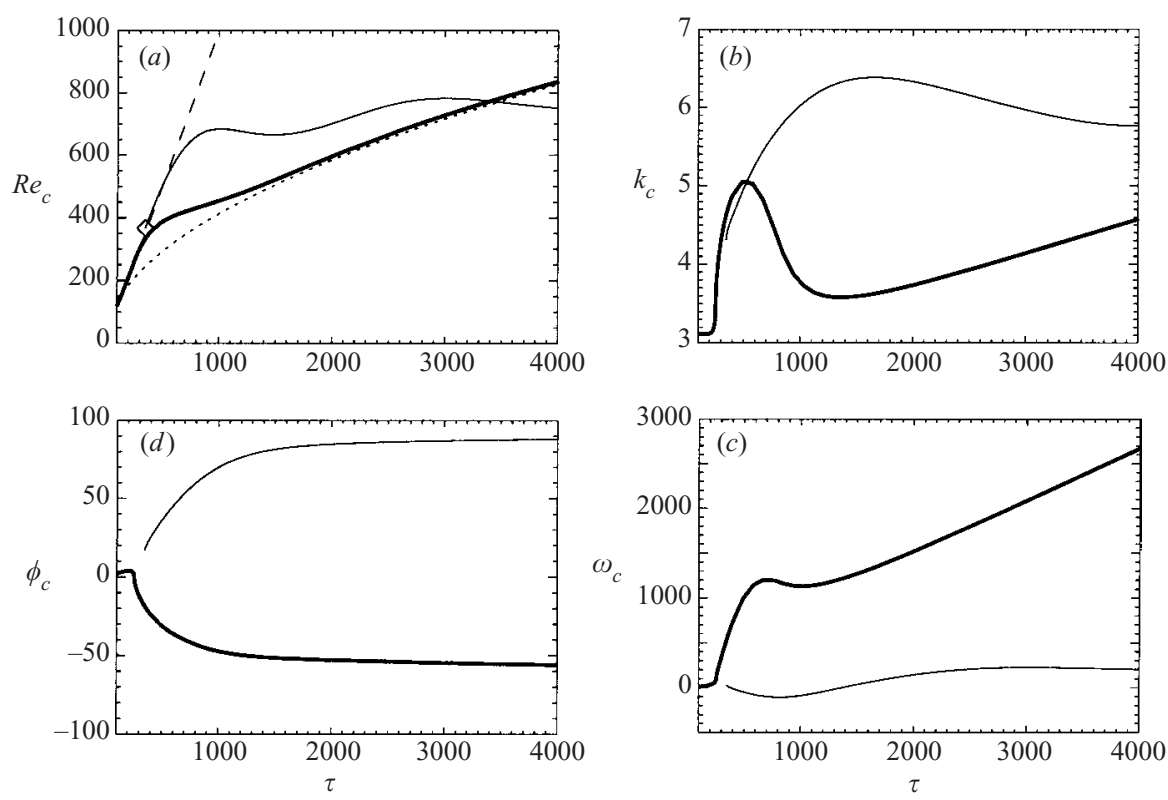

FiguRE 13. The hydrodynamic critical values for the case $R a=0$ at $\vartheta=89.5^{\circ}$, and positive $R e_{c}$. On $(a)$ the asymptotic results for type II Ekman layer instability for large $\tau \cos \vartheta$ is given by the dotted curve. The thick continuous curve, which tracks it at large $\tau \cos \vartheta$, makes a smooth transition to the Taylor vortex mode appropriate for small $\tau \cos \vartheta$. The asymptotic result for that mode is given by the long dashed curve. A new low-latitude Ekman layer mode identified by the thin continuous curve begins at the hollow diamond.

a manifestation of this difference in the models, which becomes more important at moderately small values of $\tau$.

The most intriguing feature of the $\vartheta=89.5^{\circ}$ results portrayed in figure 13 is that at $\tau \approx 3410$ the low-latitude Ekman layer mode is the preferred mode of instability. Even at such large $\tau$, the value of $\mu$ is only 3.86, indicating that we have not yet reached the true asymptotic regime. Consequently we cannot say which mode is preferred as $\tau \rightarrow \infty$. We must therefore add the caveat that, in the large- $\tau$ limit, low-latitude Ekman layer modes may be preferred for $\vartheta$ very close to $90^{\circ}$. Nevertheless, since this is a small and rather extreme parameter range, in which it is difficult to perform accurate and reliable computations, we pursue the matter no further.

\subsection{Convective instability: $\operatorname{Pr}=1, R a \neq 0$}

We now reintroduce convection, and take the angle of rotation to be $\vartheta=\pi / 4$, corresponding to mid-latitudes, with $P r=1$. We plot the stability characteristics as functions of $\tau$ for positive Reynolds number $R e=300$ in figure 14 and for negative Reynolds number $R e=-800$ in figure 15 . The general features are not dissimilar to the case of vertical rotation. Nevertheless, there are subtle differences in detail, which stem from the asymmetries that arise from the presence of the horizontal component of rotation $\boldsymbol{\Omega}_{h}$. Perhaps, the most striking difference is that the small- $\tau$ convective mode branch $\mathrm{C}_{\mathrm{S}}$ is disconnected from the large- $\tau$ convective mode branch $\mathrm{C}_{\mathrm{L}}$ in both cases that we considered (see figures 14, 15).

The case of positive $U_{0}$ with $R e=300$ is illustrated in figure 14. Significantly, there are two values of $\tau$, roughly 40 and 210 , at which $R a_{c}$ vanishes. These two values are determined by the intersection of the line $R e_{c}=300$ with the (solid) type II 

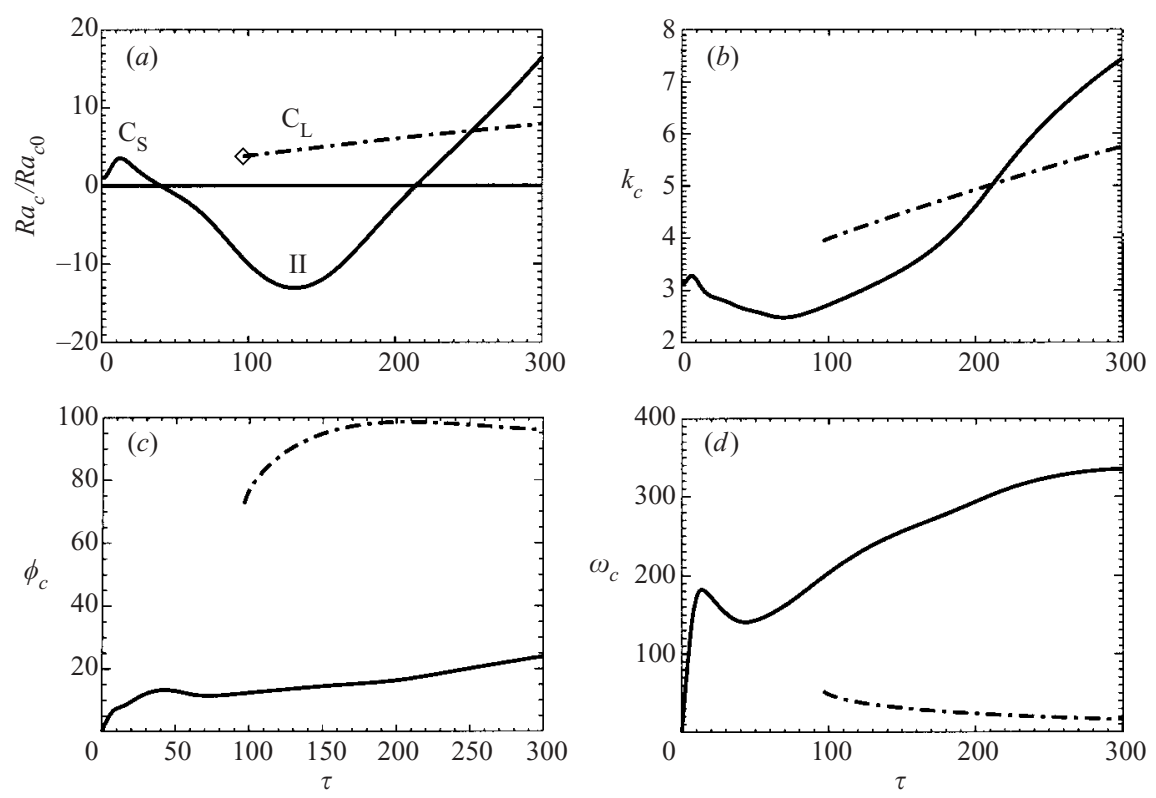

FIgURE 14. As in figure 5 but for oblique rotation $\vartheta=\pi / 4, \operatorname{Pr}=1$ and positive Reynolds number $R e=300$. The type $C_{S}$ and II modes merge continuously and are identified by the solid lines. The type $C_{L}$ mode is identified by the dot-dashed lines and its associated local minimum disappears approximately at the location of the hollow diamonds.

curve on figure 11(a) (the type I mode does not appear in figure 14 for the ranges of $R a_{c}$ and $\tau$ shown). This scenario is similar to the vertical rotation case illustrated in figure 5. Unlike the vertically rotating case, however, on decreasing $\tau$ the minimum merges smoothly with the small- $\tau$ convective mode $C_{S}$, while the large- $\tau$ mode $C_{L}$ is disconnected from it. The change in preference between type II and convective $\mathrm{C}_{\mathrm{L}}$ modes occurs at $\tau \simeq 252$. The results of the following $\S 4.3$ predict that $\phi_{c} \downarrow \pi / 2$ and $\omega_{c} \downarrow 0$ as $\tau \rightarrow \infty$ (see $(4.8 a, b)$ ), provided that $\tau^{2 / 3} \gg R e$. These trends are evident in figure 14(c,d) though even at the right of those plots we have not yet achieved the asymptotic limit.

The case of negative $U_{0}$ with $R e=-800$ is illustrated in figure 15. Again there are two values of $\tau$, now roughly 30 and 80 , at which $R a_{c}$ vanishes. These two values are determined by the intersection of the line $R e_{c}=-800$ with the (dotted) type I curve on figure $11(b)$. On the somewhat wider range roughly $20 \leqslant \tau \leqslant 80$ in figure $15(a)$, the type I mode is the preferred minimum. Though figure 11(b) suggests that there might be a $\tau$-range over which the type II mode is the preferred minimum, that is never the case as figure 15(a) shows. On increasing $\tau$ the convective mode minimum $\mathrm{C}_{\mathrm{S}}$ eventually disappears at $\tau \simeq 307$. Before that, however, the preferred minimum changes to a convective $C_{L}$ mode at $\tau \simeq 220$. As $\tau \rightarrow \infty$, the asymptotic formulae $(4.8 a, b)$ again hold for the $\mathrm{C}_{\mathrm{L}}$ mode. Though the trend $\omega_{c} \uparrow 0$ is evident in figure $15(d)$, the value of $\phi_{c}$ illustrated in figure $15(c)$ continues to increase monotonically towards $\pi / 2$, a behaviour which does not conform with the asymptotic prediction $\phi_{c} \downarrow \pi / 2$. This lack of agreement even at $\tau=400$ on the right of the figure can be traced to the large Reynolds number $R e=-800$, which moves the asymptotic regime to much larger values of $\tau$. So to confirm the validity of the large- $\tau$ analysis, figure 16 shows the $C_{L}$ mode branch followed as far as $\tau=10^{5}$. We observe in figure 16(c) that the 

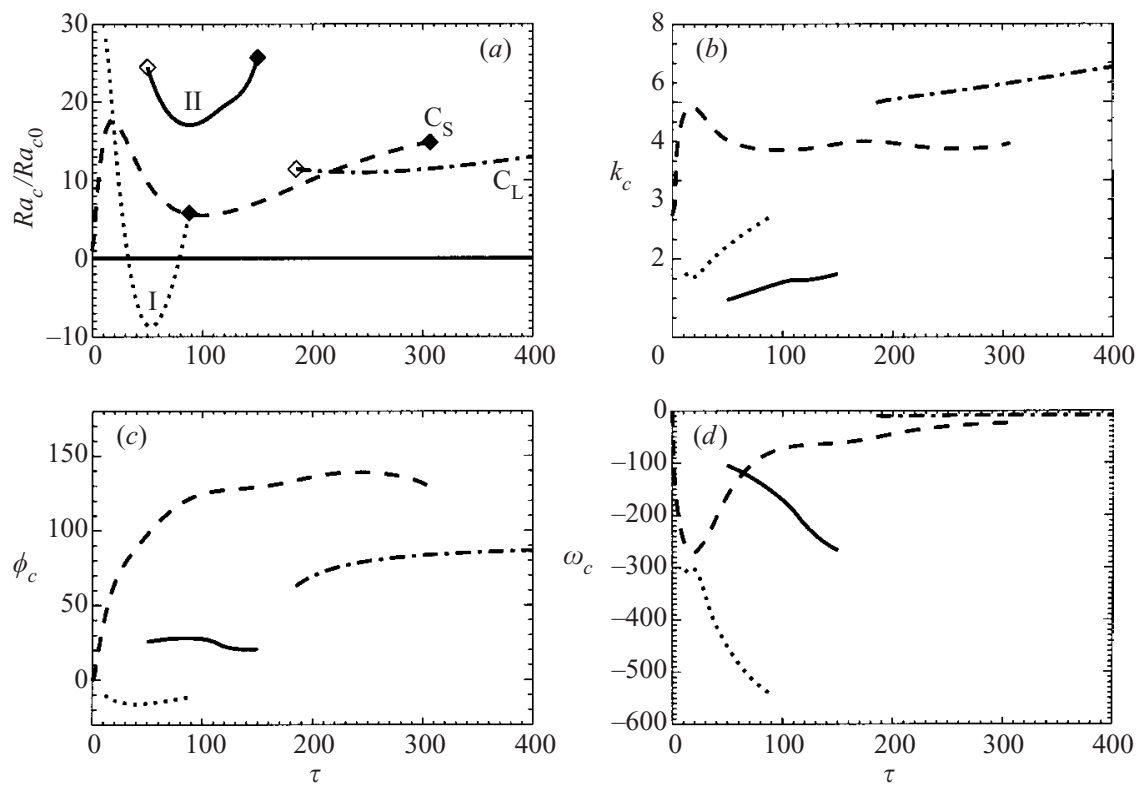

Figure 15. As in figure 14 but for oblique rotation $\vartheta=\pi / 4, \operatorname{Pr}=1$ and negative Reynolds number $R e=-800$. The type $\mathrm{C}_{\mathrm{S}}$, I, II and $\mathrm{C}_{\mathrm{L}}$ modes are identified by the dashed, dotted, continuous and dot-dashed lines respectively. The approximate left (right) locations of the disappearance of the local minima are marked by the hollow (solid) diamonds.
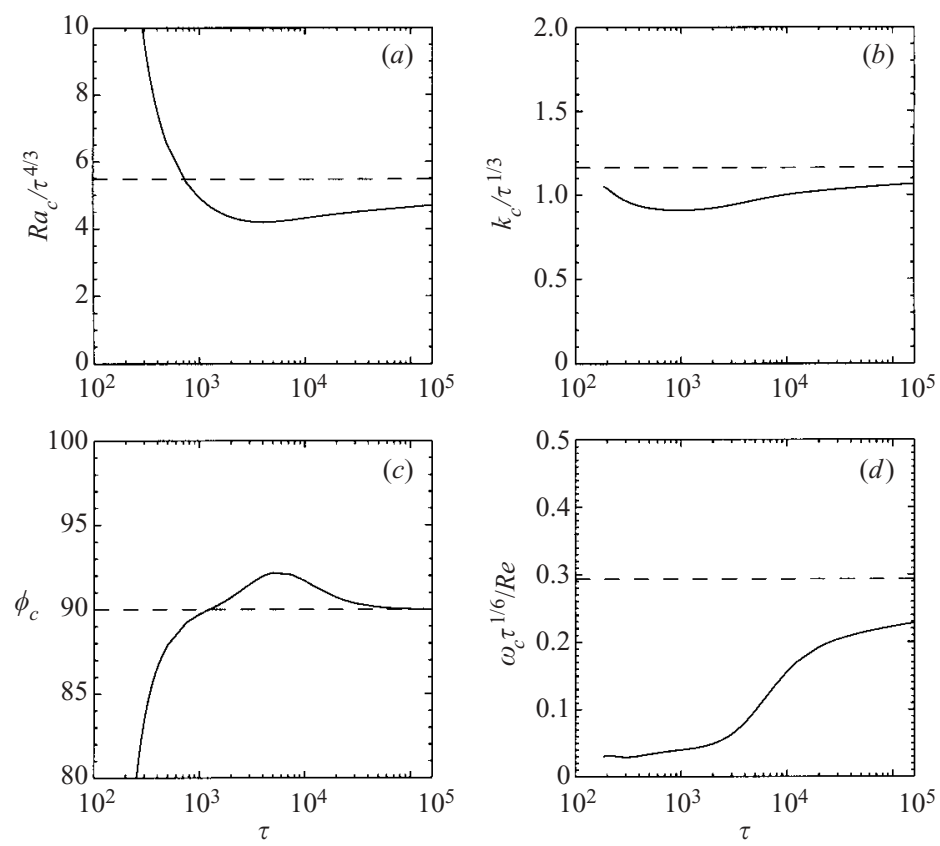

FIGURE 16. The critical values for the $C_{L}$ branch with oblique rotation $\vartheta=\pi / 4, P r=1$, plotted against $\tau$ on a logarithmic scale. The cases illustrated are $R e=-800$ (solid) and the asymptotic critical values (dashed). (a) The normalized critical Rayleigh number $R a_{c} / \tau^{4 / 3}$, (b) the normalized critical wavenumber $k_{c} / \tau^{1 / 3},(c)$ the preferred orientation $\phi_{c}$, and $(d)$ the normalized frequency $\omega_{c} \tau^{1 / 6} / R e$. 
angle $\phi_{c}$ first overshoots $\pi / 2$ and then drops back to $\pi / 2$ in the correct asymptotic manner (see (4.3), (4.8a)), when $\tau$ is about $10^{4}$. Also shown on figure $16(a, b, d)$ are the other parameters $R a_{c}, k_{c}$ and $\omega_{c}$, scaled with powers of $R e$ and $\tau$ identified in the figure caption and chosen to test the asymptotic limits (constants on the figures) predicted by $(4.6 a, b)$ and $(4.8 b)$. The agreement with theory is very satisfactory.

\subsection{Large rotation rate $\tau \gg 1, \tau^{2 / 3} \gg R e$}

Here we sketch the asymptotic analysis for the case of rapid rotation with oblique rotation $\vartheta \neq 0$. In this limit, the roll axis attempts to align itself northwards with the horizontal component of the rotation axis. Nevertheless, the Ekman layer effect studied in $\S 3.3$ and the Appendix provides a mechanism for non-alignment. Therefore we write

$$
\phi=\frac{1}{2} \pi+\Phi, \quad \text { where } \quad|\Phi| \ll 1,
$$

with the objective of determining the small critical angle $\Phi=\Phi_{c}$, which minimizes $R a$, and the corresponding small critical frequency $\omega_{c}$.

To that end, we note that in our large- $\tau$, small- $\Phi$ limit the main-stream solution that replaces (3.3) is

$$
\Psi \sim \bar{\Psi} \exp [\mathrm{i}(\tan \vartheta)(k \Phi) z] \sin l(z-\bar{z}), \quad \text { where } \quad k=O\left(\tau^{1 / 3}\right),
$$

with similar expressions for $V$ and $\Theta$. In this way the complete solution (2.20) becomes almost independent of the coordinate parallel to the rotation axis even when $k \Phi$ itself is large. Accordingly the rolls are inclined to the vertical and so enhance the dissipation. This is manifested in $(2.21 c, d)$ by the approximation $\widetilde{\Delta} \sim$ $-\left[1+(\tan \vartheta)^{2} \Phi^{2}\right] k^{2}$ provided that $k \Phi \gg 1$. At very lowest order we ignore boundary layer effects and solve the main-stream problem subject to the boundary conditions $\Psi=0$ on $z=0$ and 1 . They yield $\bar{z}=0$ and $l=\pi$. All these approximations indicate that the equation which replaces (3.4) at leading order is

$$
k^{2} R a \sim\left[1+(\tan \vartheta)^{2} \Phi^{2}\right]^{3} k^{6}+(\pi \tau \cos \vartheta)^{2} .
$$

Here, the quadratic dependence on $\Phi$ illustrates the fact that the critical values of the Rayleigh number and wavenumber, namely

$$
R a_{c} \sim 3\left(\pi^{2} / 2\right)^{2 / 3}(\tau \cos \vartheta)^{4 / 3}, \quad k_{c} \sim(\pi \tau \cos \vartheta / \sqrt{2})^{1 / 3},
$$

occur close to $\Phi=0$.

The incorporation of the boundary layer jump conditions requires care but the upshot of the analysis is that $l$ continues to be given by (3.6b). Consequently equations $(3.7 a, b)$ continue to hold with $\tau$ replaced by $\tau \cos \vartheta$ and with the additional $\Phi$ dependence identified in (4.5). Specifically, at leading order (3.7a) becomes

$$
R a \sim \frac{(\tau \cos \vartheta)^{2}}{k^{2}}\left\{\pi^{2}+\frac{k^{6}}{(\tau \cos \vartheta)^{2}}\left[1+3(\tan \vartheta)^{2} \Phi^{2}\right]-\frac{2 \pi^{2}(\tau \cos \vartheta)^{1 / 2}}{k^{2}} \operatorname{Re}\{\Gamma\}\right\} .
$$

Using (A 3b), (A 7c) and (A 14c), minimization of $R a$ over small $\Phi$ determines the critical angle

$$
\Phi_{c}=\frac{29}{2}\left(\frac{R e}{30 \tan \vartheta}\right)^{2}\left(\frac{2}{\tau \cos \vartheta}\right)^{3 / 2}
$$


The corresponding critical frequency, determined by (3.7b) and (A 11c) on the basis that $\phi_{c} \approx \pi / 2$, is

$$
\operatorname{Pr}^{-1} \omega_{c} \sim \frac{3 \operatorname{Re}}{10(3 \operatorname{Pr}-1)}\left(\frac{4 \pi^{2}}{\tau \cos \vartheta}\right)^{1 / 6} \quad\left(\operatorname{Pr} \neq \frac{1}{3}\right) .
$$

It should be emphasized that though we derived (4.5) in the limit $k \Phi \gg 1$, the expressions (4.5) and (4.7) capture the correct $\Phi$ dependence for all values of $k \Phi$, even as $\Phi$ decreases to zero. This fact ensures that $(4.8 a)$ holds as $\tau \rightarrow \infty$. Thus the remaining requirement $\Phi_{c} \ll 1$ is met provided that $\tau^{3 / 4} \gg R e$. Neverthless, that inequality is automatically satisfied when $\tau^{2 / 3} \gg R e$, which is necessary for the validity of the boundary layer calculation.

Finally we remark that the symmetries under the interchange $R e \leftrightarrow-R e$ (i.e. $U_{0} \leftrightarrow$ $-U_{0}$ ) implied by (4.8) are simply consequences of our low-order theory and are not exhibited by the actual solution.

\section{Discussion}

In our study the nature of the convective instability is affected by three different physical ingredients. They are the magnitudes of the vertical and horizontal components $\boldsymbol{\Omega}_{v}$ and $\boldsymbol{\Omega}_{h}$ of the rotation vector, and the horizontal shear $\boldsymbol{\Lambda}$ driven by the bottom boundary velocity $\boldsymbol{U}_{0}$. Provided that $\left|\boldsymbol{\Omega}_{v}\right|$ is sufficiently large an Ekman layer forms on the lower boundary. We have explored the ensuing mode competition between hydrodynamic and convective instabilities that occurs in the presence of buoyancy forces; in the absence of rotation such competition has been investigated by Fujimura \& Kelly (1988) and Mohamad \& Viskanta (1989). Our study has been complicated by the fact that the marginal stability surface defined by the neutral Rayleigh number $R a=R a(k, \phi)$ generally has multiple minima. These may include the type I and II hydrodynamic mode minima as well as the convective minimum. These minima can emerge and disappear in the $(k, \phi)$-plane as figure 6 illustrates.

When there is a horizontal component of rotation $\left(\left|\boldsymbol{\Omega}_{h}\right| \neq 0\right)$, the stability characteristics are sensitive to the sign of $\boldsymbol{U}_{0}$. For positive $\operatorname{Re}\left(\boldsymbol{U}_{0}\right.$ pointing westwards) the critical Rayleigh number $R a_{c}$ is generally smaller than for negative $\operatorname{Re}\left(\boldsymbol{U}_{0}\right.$ pointing eastwards); similar behaviour was identified by Cox (1998). Likewise the magnitude $\left|R e_{c}\right|$ of the critical Reynolds numbers in the absence of buoyancy forces at given colatitude $\vartheta$ is generally smaller for positive $R e$. This feature is also evident in figure 2 of Hoffmann \& Busse (1999), in which their $\gamma$ is the co-latitude. The difference is accentuated as $\left|\boldsymbol{\Omega}_{v}\right|$ is reduced to zero. Indeed, in the plane Couette flow limit of horizontal rotation $(\vartheta=\pi / 2)$, the pure hydrodynamic Taylor vortex instability $(R a=0)$ is only possible for $R e>0$. These asymmetries of the stability characteristics may have Solar implications, for, according to the interpretation of helioseismological results, the differential rotation of the convection zone is slower (faster) near the pole (equator) than that of the radiative zone below it. So relative to local axes fixed in the differentially rotating convection zone, the tachocline shear layer is characterized by $R e>0(R e<0)$ near the pole (equator).

The dynamo properties of the flows described here have been investigated by Ponty et al. $(2001 a, b)$. Of particular interest are the large-Taylor-number $\left(\tau^{2} \gg 1\right)$ convective modes as illustrated for the case $\tau=200$ in figure 9. Though $\tau$ there is not particularly large, it does illustrate the point that most of the convection takes place outside the shear layer with a small part overshooting into it. Since the shear in the layer is quite strong, it opens up the possibility of mixing between 
one cell and its neighbours through horizontal transport in the Ekman layer. This has important implications with respect to the question of possible fast dynamo mechanisms operating in the tachocline.

Yannick Ponty gratefully acknowledges support from the Leverhulme Trust under Research Grant F/144/AH. We all acknowledge the support of the CNRS/Royal Society cooperation agreement (2002-2003). Andrew Soward thanks the Observatory of the Côte d'Azur for their kind hospitality during two visits 31 March to 7 April 2001 and 13 to 19 April 2002. We have benefited from valuable discussions with Professors F. H. Busse, C. A. Jones, K. Zhang and Dr P. C. Matthews.

The numerical calculations were performed using the computing facilities of the parallel computer Ceres at the University of Exeter, the computing facilities of the laboratory Cassini, Observatory of the Côte d'Azur, provided by the program 'Simulations Interactives et Visualisation en Astronomie et Mécanique (SIVAM)', and the computing facilities of IDRIS (Palaiseau, France).

\section{Appendix. The Ekman layer}

In the large- $\tau$ limit, the buoyancy force in the bottom Ekman layer is negligible. Thus motion there is governed by the first two components of $(2.21 a)$, which reduce under the boundary layer approximations to

$$
\begin{gathered}
U^{\prime \prime}+\tau\left(V-V_{\infty}\right)=\mathrm{i} k \operatorname{Re}\left(\lambda_{1} U+\lambda_{1}^{\prime} \Psi\right), \\
V^{\prime \prime}-\tau U=\mathrm{i} k \operatorname{Re}\left(\lambda_{1} V+\lambda_{2}^{\prime} \Psi\right),
\end{gathered}
$$

where $V_{\infty}=V\left(0_{+}\right)$in (3.5) and

$$
\Psi=-\int_{0}^{z} U \mathrm{~d} z
$$

(cf. (2.16)). The boundary conditions are

$$
U=0, \quad V=0 \quad \text { on } \quad z=0 .
$$

The solution with $(U, V) \rightarrow\left(0, V_{\infty}\right)$ on leaving the Ekman layer leads to the relation

$$
\Psi \rightarrow \tau^{-1 / 2} \Gamma_{B} V_{\infty} \quad \text { as } \quad \tau^{1 / 2} z \rightarrow \infty,
$$

in which we will determine $\Gamma_{B}$ as a function of $\phi$ and

$$
\Delta=\mathrm{i} \frac{k \operatorname{Re}}{\tau} \text {. }
$$

A compact representation of the solution can be obtained by writing

$$
U+\mathrm{i}\left(V-V_{\infty}\right)=V_{\infty} u(\zeta), \quad \text { where } \quad \zeta=\tau^{1 / 2} z
$$

Then we have

$$
U=V_{\infty}\left(u+u^{*}\right) / 2, \quad V=V_{\infty}\left(u-u^{*}\right) /(2 \mathrm{i})+V_{\infty},
$$

where the star denotes the complex conjugate taken when $\Delta$ is real. Accordingly $u$ satisfies

where

$$
u_{, \zeta \zeta}-\beta^{2} u=F(\Delta, \zeta), \quad u(0)=-\mathrm{i} \quad\left(\beta=\mathrm{e}^{\mathrm{i} \pi / 4}\right),
$$

$$
F(\Delta, \zeta)=\Delta\left[\operatorname{Re}\{\lambda\}(u+\mathrm{i})+\lambda_{, \zeta} \psi\right], \quad \lambda=-\mathrm{i} \mathrm{e}^{\mathrm{i} \phi} \mathrm{e}^{-\beta \zeta}
$$


and

$$
\psi(\zeta)=-\frac{1}{2} \int_{0}^{\zeta}\left[u(\xi)+u^{*}(\xi)\right] \mathrm{d} \xi \quad \text { giving also } \quad \Gamma_{B}=\psi(\infty)
$$

As an aid to the determination of $\Gamma_{B}$, we note that the Green's function solution

$$
u(\zeta)=-\mathrm{i} \mathrm{e}^{-\beta \zeta}+\mathrm{i} \beta\left[\mathrm{e}^{-\beta \zeta} \int_{0}^{\zeta} \sinh \beta \xi F(\Delta, \xi) \mathrm{d} \xi+\sinh \beta \zeta \int_{\zeta}^{\infty} \mathrm{e}^{-\beta \xi} F(\Delta, \xi) \mathrm{d} \xi\right]
$$

of (A $6 a$ ) may be used to establish the identity

$$
\int_{0}^{\infty} u \mathrm{~d} \zeta=-\beta+\mathrm{i} \int_{0}^{\infty}\left(1-\mathrm{e}^{-\beta \zeta}\right) F(\Delta, \zeta) \mathrm{d} \zeta
$$

We emphasize again that the star denotes the complex conjugate taken when $\Delta$ is real; the required results for imaginary $\Delta$ can only be obtained by analytic continuation.

In the small- $|\Delta|$ limit, we may seek a series expansion

$$
\begin{gathered}
u=u_{0}+\Delta u_{1}+\Delta^{2} u_{2}+\ldots, \quad \psi=\psi_{0}+\Delta \psi_{1}+\Delta^{2} \psi_{2}+\ldots, \\
\Gamma_{B}=\Gamma_{0}+\Delta \Gamma_{1}+\Delta^{2} \Gamma_{2}+\ldots, \quad F=\Delta F_{1}+\Delta^{2} F_{2}+\ldots
\end{gathered}
$$

Thus at lowest order (A $6 d, e, g$ ) determine trivially

$$
\Gamma_{0}=1 / \sqrt{2}
$$

while from (A $6 a) u_{0}$ satisfies

$$
u_{0, \zeta \zeta}-\mathrm{i} u_{0}=0 \quad \text { with } \quad u_{0}(0)=-\mathrm{i} .
$$

The required solution, which tends to zero at infinity, is

$$
u_{0}=-\mathrm{i} \mathrm{e}^{-\beta \zeta}, \quad \psi_{0}=\operatorname{Re}\left\{\beta\left(1-\mathrm{e}^{-\beta \zeta}\right)\right\} .
$$

At $O(|\Delta|)$ we have

$$
F_{1}=\operatorname{Re}\{\lambda\}\left(u_{0}+\mathrm{i}\right)+\lambda_{, \zeta} \psi_{0}
$$

which using (A $10 a, b)$ is

$$
F_{1}=\frac{1}{2}\left[-\mathrm{i}\left(\mathrm{e}^{\mathrm{i} \phi}+\mathrm{ie}^{-\mathrm{i} \phi}\right) \mathrm{e}^{-\sqrt{2} \zeta}-\mathrm{e}^{-\mathrm{i} \phi} \mathrm{e}^{-\beta^{*} \zeta}+\mathrm{ie}^{\mathrm{i} \phi} \mathrm{e}^{-\beta \zeta}\right] .
$$

Accordingly (A $6 d, e, g$ ) determine

$$
\Gamma_{1}(\phi)=\frac{-7 \cos \phi+3 \sin \phi}{20 \sqrt{2}} .
$$

Furthermore by (A 6a) $u_{1}$ satisfies

$$
u_{1, \zeta \zeta}-\mathrm{i} u_{1}=F_{1} \quad \text { with } \quad u_{1}(0)=0
$$

from which we obtain

$$
\begin{aligned}
u_{1}=\frac{1}{20}\left\{2(1-2 \mathrm{i})\left(\mathrm{e}^{\mathrm{i} \phi}+\mathrm{ie}^{-\mathrm{i} \phi}\right) \mathrm{e}^{-\sqrt{2} \zeta}-5 \mathrm{ie}^{-\mathrm{i} \phi} \mathrm{e}^{-\beta^{*} \zeta}\right. \\
\left.-\left[5 \beta \mathrm{e}^{\mathrm{i} \phi} \zeta+\left(2(1-2 \mathrm{i}) \mathrm{e}^{\mathrm{i} \phi}+(4-3 \mathrm{i}) \mathrm{e}^{-\mathrm{i} \phi}\right)\right] \mathrm{e}^{-\beta \zeta}\right\}, \\
\psi_{1}=\frac{1}{20} \operatorname{Re}\left\{-(5-2 \mathrm{i}) \beta \mathrm{e}^{\mathrm{i} \phi}+6 \beta \mathrm{e}^{-\mathrm{i} \phi} \mathrm{e}^{-\sqrt{2} \zeta}\right. \\
\left.-\left[5 \mathrm{e}^{\mathrm{i} \phi} \zeta-\beta\left((9+7 \mathrm{i}) \mathrm{e}^{\mathrm{i} \phi}+(3+4 \mathrm{i}) \mathrm{e}^{-\mathrm{i} \phi}\right)\right] \mathrm{e}^{-\beta \zeta}\right\} .
\end{aligned}
$$


At $O\left(|\Delta|^{2}\right)$ we have

which using (A 13a,b) is

$$
F_{2}=\operatorname{Re}\{\lambda\} u_{1}+\lambda_{, \zeta} \psi_{1},
$$

$$
\begin{aligned}
F_{2}= & \frac{1}{40}\left\{\left[-10 \mathrm{i} \beta \zeta+(4+3 \mathrm{i}) \mathrm{e}^{2 \mathrm{i} \phi}-(2-7 \mathrm{i})-(3+4 \mathrm{i}) \mathrm{e}^{-2 \mathrm{i} \phi}\right] \mathrm{e}^{-\sqrt{2} \zeta}\right. \\
& -4(1+\mathrm{i})\left(-\mathrm{ie}^{2 \mathrm{i} \phi}+1\right) \mathrm{e}^{-(\beta+\sqrt{2}) \zeta}+2(2+\mathrm{i})\left(1+\mathrm{ie}^{-2 \mathrm{i} \phi}\right) \mathrm{e}^{-\left(\beta^{*}+\sqrt{2}\right) \zeta} \\
& \left.-5(1+\mathrm{i}) \mathrm{e}^{2 \mathrm{i} \phi} \mathrm{e}^{-2 \beta \zeta}+5 \mathrm{e}^{-2 \mathrm{i} \phi} \mathrm{e}^{-2 \beta^{*} \zeta}+\left[(5-2 \mathrm{i}) \mathrm{e}^{2 \mathrm{i} \phi}+(2-5 \mathrm{i})\right] \mathrm{e}^{-\beta \zeta}\right\} .
\end{aligned}
$$

Accordingly (A $6 d, e, g$ ) determine

$$
\Gamma_{2}(\phi)=\frac{-21 \cos 2 \phi+29 \sin 2 \phi}{600 \sqrt{2}}+\text { constant, }
$$

where the value of the constant is of no interest to us.

The main and important point is that

$$
\operatorname{Re}\left\{\Gamma_{B}\right\} \sim \Gamma_{0}-\left(\frac{k R e}{\tau}\right)^{2} \Gamma_{2}
$$

is maximized where $\Gamma_{2}$ is minimized at

$$
\phi_{c}=-\frac{1}{2} \tan ^{-1} \frac{29}{21} \approx-27.0451^{\circ} \text {. }
$$

This selects the orientation $\phi_{c}$ of the rolls which minimizes the Rayleigh number (3.7a), so determining its critical value as a perturbation of $(3.8 a)$. The corresponding value of

is determined by

$$
\operatorname{Im}\left\{\Gamma_{B}\right\} \sim \frac{k \operatorname{Re}}{\tau} \Gamma_{1}
$$

$$
\Gamma_{1}\left(\phi_{c}\right) \approx-0.268652,
$$

which in turn fixes the critical frequency (3.11).

\section{REFERENCES}

AsaI, T. \& NAKASUJI, I. 1973 On the stability of Ekman boundary layer flow with thermally unstable stratification. J. Met. Soc. Japan 51, 29-42.

Brown, R. A. 1972 On the inflexion point instability of a stratified Ekman boundary layer. J. Atmos. Sci. 29, 850-859.

Busse, F. H. \& KRopP, M. 1992 Buoyancy driven instabilities in rotating layers with parallel axis of rotation. Z. Angew. Math. Phys. 43, 28-35.

Caldwell, D. R. \& Van Atta, C. W. 1970 Characteristics of Ekman boundary layer instabilities. J. Fluid. Mech. 44, 79-95.

Clever, R. M., Busse, F. H. \& Kelly, R. E. 1977 Instabilities of longitudinal convection rolls in Couette flow. Z. Angew. Math. Phys. 28, 771-783.

Cox, S. M. 1998 Rotating convection in a shear flow. Proc. R. Soc. Lond. A 454, 1699-1717.

Dziembowski, W., Goode, P. R. \& LibBrecht, K. G. 1989 The radial gradient in the Sun's rotation. Astrophys. J. 337, L53-L57.

ETLing, D. 1971 The stability of an Ekman boundary layer flow as influenced by the thermal stratification. Beitr. Phys. Atmosph. 44, 168-186.

ETLING, D. \& BRown, R. A. 1993 Roll vortices in the planetary boundary: a review. Boundary-Layer Met. 65, 215-248.

FALLER, A. J. 1963 An experimental study of the instability of the laminar Ekman boundary layer. J. Fluid Mech. 15, 560-576. 
Faller, A. J. \& KaYlor, R. E. 1966 A numerical study of the instability of laminar Ekman boundary layer flow. J. Atmos. Sci. 23, 466-480.

Fujimura, K. \& Kelly, R. E. 1988 Stability of unstably stratified shear flow between parallel plates. Fluid Dyn. Res. 2, 281-292.

GAGE, K. S. \& REID, W. H. 1968 The stability of thermally stratified plane Poiseuille flow. J. Fluid Mech. 33, 21-32.

Gottlieb, D. \& Orszag. S. A. 1977 Numerical Analysis of Spectral Methods: Theory and Applications. SIAM, Philadelphia.

Greenspan, H. P. 1968 The Theory of Rotating Fluids. Cambridge University Press.

Hathaway, D. H. \& Somerville, R. C. J. 1986 Nonlinear interactions between convection, rotation and flows with vertical shear. J. Fluid Mech. 164, 91-105.

Hathaway, D. H., Toomre, J. \& Gilman, P. A. 1980 Convective instability when the temperature gradient and rotation vector are oblique to gravity. II. Real fluids with effects of diffusion. Geophys. Astrophys. Fluid Dyn. 15, 7-37.

Hoffman, N. \& Busse, F. H. 1999 Instabilities of shear flows between two coaxial differentially rotating cones. Phys. Fluids 11, 1676-1678.

Hoffman, N. \& Busse, F. H. 2001 Linear instabilities of Poiseuille-Couette-Ekman flows: Local results for flows between differentially rotating disks with throughflow. Phys. Fluids 13, 27352738.

Hoffman, N., Busse, F. H. \& Chen, W.-L. 1998 Transition to complex flows in the Ekman-Couette layer. J. Fluid Mech. 366, 311-331.

Ingersoll, A. P. 1965 Convective instabilities in plane Couette flow. Phys. Fluids 9, 682-689.

Iooss, G., Nielsen, H. B. \& True, H. 1978 Bifurcation of the stationary Ekman flow into a stable periodic flow. Arch. Rat. Mech. Anal. 68, 227-256.

KelLy, R. E. 1994 The onset and development of thermal convection in fully developed shear flows. Adv. Appl. Mech. 31, 35-112.

Kropp, K. \& Busse, F. H. 1991 Thermal convection in differentially rotating systems. Geophys. Astrophys. Fluid Dyn. 61, 127-148.

Leibovich, S. \& Lele, S. K. 1985 The influence of the horizontal compoment of Earth's angular velocity on the instability of the Ekman layer. J. Fluid Mech. 150, 41-87.

Lilly, D. K. 1966 On the instability of Ekman boundary flow. J. Atmos. Sci. 23, 481-494.

Matthews, P. C. \& Cox, S. 1997 Linear stability of rotating convection in an imposed shear flow. J. Fluid Mech. 350, 271-293.

Melander, M. V. 1983 An algorithmic approach to the linear stability of the Ekman layer. J. Fluid Mech. 132, 283-293.

Mohamad, A. A. \& Viskanta, R. 1989 Stability of a lid-driven shallow cavity heated from below. Intl J. Heat Mass Transfer 32, 2155-2166.

Ponty, Y., Gilbert, A. D. \& Soward, A. M. $2001 a$ Kinematic dynamo action in large magnetic Reynolds number flows driven by shear and convection. J. Fluid Mech. 435, 261-287.

Ponty, Y., Gilbert, A. D. \& Soward, A. M. $2001 b$ Dynamo action due to Ekman layer instability. In Dynamo and Dynamics, a Mathematical Challenge (ed. P. Chossat, D. Armbruster \& I. Oprea). NATO ASI, pp. 75-82. Kluwer.

Spiegel, E. A. \& ZAHn, J.-P. 1992 The Solar tachocline. Astron. Astrophys. 265, 106-114.

Weiss, N. O. 1994 Solar and stellar dynamos. In Lectures on Solar and Planetary Dynamos (ed. M. R. E. Proctor \& A. D. Gilbert), pp. 59-95. Cambridge University Press.

Wippermann, F. K., Etling, D. \& Kirstein, H. J. 1978 On the instability of a planetary boundary layer with Rossby number similarity. Boundary-Layer Met. 15, 301-321. 